\title{
Adaptive Fuzzy Dynamic Surface Sliding Mode Position Control for a Robot Manipulator with Friction and Deadzone
}

\author{
Jeong Yun Cheong, Seong Ik Han, and Jang Myung Lee \\ Department of Electronic Engineering, Pusan National University, Jangjeon-dong, Geumjeong-gu, Busan 609-735, Republic of Korea \\ Correspondence should be addressed to Jang Myung Lee; jmlee@pusan.ac.kr
}

Received 23 July 2013; Revised 20 October 2013; Accepted 20 October 2013

Academic Editor: Xudong Zhao

Copyright (C) 2013 Jeong Yun Cheong et al. This is an open access article distributed under the Creative Commons Attribution License, which permits unrestricted use, distribution, and reproduction in any medium, provided the original work is properly cited.

Precise tracking positioning performance in the presence of both the deadzone and friction of a robot manipulator actuator is difficult to achieve by traditional control methodology without proper nonlinear compensation schemes. In this paper, we present a dynamic surface sliding mode control scheme combined with an adaptive fuzzy system, state observer, and parameter estimator to estimate the uncertainty, friction, and deadzone nonlinearities of a robot manipulator system. We design a dynamic surface sliding mode basic controller by systematic recursive design steps that yields several adaptive laws for the compensation of nonlinear friction, deadzone, and other unknown nonlinear dynamics. The boundedness and convergence of this closed-loop system are guaranteed by the Lyapunov stability theorem. Experiments on the Scorbot robot manipulator demonstrate the validity and effectiveness of the proposed control scheme.

\section{Introduction}

In recent decades, several advanced control approaches have been developed to solve complex control problems as industrial machines and devices have rapidly progressed, requiring higher performance control. Among these, a breakthrough nonlinear control method, adaptive backstepping, $[1,2]$ achieved stabilizing controllers for nonlinear system and guaranteed global or regional regulation and tracking properties. The cancellation of useful nonlinearities that occur with the feedback linearization techniques can be also avoided by using a step-by-step recursive algorithm. However, the application of the backstepping design method requires that nonlinear dynamic models be known either exactly or linearly parameterized with respect to known nonlinear functions. In real situations, this requirement is frequently difficult to accomplish since most uncertainty in a nonlinear system is unknown. To solve this problem, adaptive backstepping methods combined with fuzzy methods $[3,4]$ and neural networks (NNs) $[5,6]$ have been developed to approximate these unknown uncertainties. Thus, recently, this approximator-based backstepping method has become a very popular control scheme for dealing with a large class of nonlinear systems. However, repeated differentiation of the virtual control functions [7] gives rise to an explosion of complexity in the controller terms of the complex nonlinear system. Dynamic surface control (DSC) $[7,8]$ was developed to help a nonlinear systems overcome this "explosion of terms" by using a first-order filter of the synthetic input at each step of the backstepping design procedure. Thus, several adaptive DSCs combined with fuzzy methods [9] and NNs have been developed [10-12] because these controllers are relatively much simpler than backstepping-based ones. Another option is a model-free approach such as fuzzy methods, which is synthesized.

In a dynamic system consisting of the actuator of a robot manipulator, friction and deadzones are frequently encountered and are the main obstacles to high-performance positioning and tracking control. Friction between a moving part and a guide surface gives rise to problems such as stick slip, limit cycle, and steady-state error. Deadzone nonlinearity also causes inaccuracy in a control system [13]. A controller designed to compensate for friction or deadzone independently may perform poorly in the friction/deadzone overlap. Thus, compensation for both nonlinearities should be taken into consideration together. However, with the exception 
of [12], most DSC applications have focused largely on the compensation of linear or smooth nonlinear system.

The effect of nonlinear friction appears most strongly in a low-velocity regime, especially during velocity reversals. The LuGre [14] and Elastoplastic [15] models can construct a friction estimator relatively easily by virtue of their more systematic structure and lower complexity compared to other available modes. Lin and Chen [16], Yau and Yan [17], and Han et al. $[18,19]$ developed a sliding mode control and fuzzy logic scheme with the LuGre and Elastoplastic friction model to compensate for the nonlinear friction of a ball-screw and robot systems. For deadzone, several control schemes [20-23] have been developed. However, compensations for both deadzones and friction together have not often been considered until now.

Fuzzy technology $[24,25]$ has replaced many complex nonlinear control applications. One major feature of fuzzy logic is its ability to express an amount of ambiguity, similar to judgments based on human experiences or expert opinion. Thus, fuzzy logic is an alternative way to deal with the unknown mathematical model of a complex system due to its universal approximation property [24]. A fuzzy controller depends on the experience of experts to create a fuzzy rule base and parameters that are adjusted by adaptive laws for a specified control performance. Hence, adaptive fuzzy controls have been applied successfully in many nonlinear control systems and guarantee improved system performance and stability in the Lyapunov sense [26-28]. However, a specific performance decision table, complicated learning mechanisms, and/or a large amount of fuzzy rules require design by trial-and-error and make practical application difficult.

It is well known that the sliding mode control (SMC) technique is robust to system uncertainty due to its use of a sliding surface $[29,30]$. To reduce the fuzzy rules in fuzzy control and significantly increase control performance, SMC is combined with fuzzy logic [31] and other control methods such as intelligent methods [32] and backstepping control [33]. The adaptive sliding mode backstepping control for a semistrict feedback system with unmatched uncertainty was proposed in $[34,35]$. However, the backstepping control technique has an explosion of terms problem due to the repeated differentiation of the virtual control functions. This problem leads to a severe computational burden for real hardware implementations such as complex robotic systems. Thus, although the backstepping method is theoretically tractable, in real applications, its increasing complexity is an insurmountable obstacle that prevents its application to multiple state control systems.

We propose an adaptive fuzzy strict feedback positioning control for a robot manipulator based on a DSC design. SMC is applied to a DSC and FLC frame to enhance robustness for the compensation of uncertainty and an adaptive fuzzy system approximates the unknown nonlinear function. The main contributions of this paper are as follows. (1) The DSC scheme is introduced to overcome the drawback of backstepping control. (2) We show that both the deadzone and friction nonsmooth and nonlinear effects of a robot manipulator can be compensated for simultaneously. (3) We then detail and show how SMC is combined with an adaptive DSC and FLC system to enhance the performance robustness for lumped uncertainty and required fuzzy rules and can then be reduced to an approximation to reduce the controller complexity. (4) The proposed control approach is successfully applied to the problem of both reducing nonsmooth nonlinear effect and uncertainty of the robot manipulator in the presence of the friction and deadzone by experiment.

\section{Problem Formulations}

2.1. Description of the Nonlinear Plant. We consider a robot manipulator system in the presence of deadzone and friction including actuator dynamics whose dynamic equations [36, 37] are described by

$$
\begin{gathered}
\mathbf{M}(\mathbf{q}) \ddot{\mathbf{q}}+\mathbf{C}(\mathbf{q}, \dot{\mathbf{q}}) \dot{\mathbf{q}}+\mathbf{G}(\mathbf{q})+\mathbf{T}_{f}(\mathbf{q}, \dot{\mathbf{q}})+\mathbf{T}_{L}=\boldsymbol{\tau}, \\
\boldsymbol{\tau}=\mathbf{n} \mathbf{k}_{t} \mathbf{i}, \\
\mathbf{L}_{m} \frac{d \mathbf{i}}{d t}+\mathbf{R}_{m} \mathbf{i}+\mathbf{k}_{b} \dot{\mathbf{q}}=\mathbf{V},
\end{gathered}
$$

where $\mathbf{q}, \dot{\mathbf{q}}, \ddot{\mathbf{q}} \in R^{n \times 1}$ denote the joint position, velocity, and acceleration vectors, respectively; the moment of inertia matrix $\mathbf{M}(\mathbf{q}) \in R^{n \times n}$ is a positive definite symmetric matrix; $\mathbf{C}(q, \dot{q}) \in R^{n \times n}$ is the centripetal Coriolis matrix; $\dot{\mathbf{M}}(\mathbf{q})-$ $2 \mathbf{C}(q, \dot{q})$ is a skew-symmetric matrix; $\mathbf{G}(\mathbf{q}) \in R^{n \times 1}$ is the gravity vector; $\mathbf{T}_{f}(\mathbf{q}, \dot{\mathbf{q}}) \in R^{n \times 1}$ is the nonlinear friction torque vector; $\mathbf{T}_{L} \in R^{n \times 1}$ is an external disturbance; $\boldsymbol{\tau} \in R^{n \times 1}$ is the deadzone control torque vector of the joint actuators; $\mathbf{i}$ is the motor current vector; $\mathbf{V}$ is the voltage vector applied to the motor drive; $\mathbf{L}_{m}$ and $\mathbf{R}_{m}$ are the inductance and resistance of the motor, respectively; and $\mathbf{k}_{b}$ is the back electromotiveforce (emf) constant of the motor.

Considering the modeling uncertainties and external disturbances, the robot system in (1) can be reformulated as

$$
\ddot{\mathbf{q}}=\mathbf{f}_{n}(\mathbf{q}, \dot{\mathbf{q}})-\mathbf{g}_{n}(\mathbf{q}) \mathbf{T}_{f}+\mathbf{g}_{n}(\mathbf{q}) \mathbf{T}_{u}+\mathbf{g}_{n}(\mathbf{q}) \boldsymbol{\tau},
$$

where the subscript $n$ represents the system parameters in the nominal condition, $\mathbf{f}_{n}(\mathbf{q}, \dot{\mathbf{q}})=-\mathbf{g}_{n}(\mathbf{q})\left[\mathbf{C}_{n}(\mathbf{q}, \dot{\mathbf{q}})+\mathbf{G}_{n}(\mathbf{q})\right]$, where $\mathbf{g}_{n}(\mathbf{q})=\mathbf{M}_{n}^{-1}(\mathbf{q}) ; \mathbf{T}_{u}$ is a lumped uncertainty defined as $\mathbf{T}_{u}=-\Delta \mathbf{M}(\mathbf{q}) \ddot{\mathbf{q}}-\Delta \mathbf{C}(\mathbf{q}, \dot{\mathbf{q}})-\Delta \mathbf{G}(\mathbf{q})-\mathbf{T}_{L} ; \Delta \mathbf{M}(\mathbf{q}), \Delta \mathbf{C}(\mathbf{q}, \dot{\mathbf{q}})$, $\Delta \mathbf{G}(\mathbf{q})$, and $\Delta \mathbf{T}_{f}$ represent the unknown uncertainties of $\mathbf{M}(\mathbf{q}), \mathbf{C}(\mathbf{q}, \dot{\mathbf{q}}), \mathbf{G}(\mathbf{q})$, and $\mathbf{T}_{f}$, respectively; and $\mathbf{T}_{L} \in R^{n \times 1}$ is the disturbance vector. The uncertainties of $\Delta \mathbf{M}(\mathbf{q}), \Delta \mathbf{C}(\mathbf{q}, \dot{\mathbf{q}})$, $\Delta \mathbf{G}(\mathbf{q})$, and $\Delta \mathbf{T}_{f}$ are bounded by some positive constants $\rho_{i}(i=m, c, g, f)$ such that $\|\Delta \mathbf{M}\| \leq \rho_{m},\|\Delta \mathbf{C}\| \leq \rho_{c},\|\Delta \mathbf{G}\| \leq$ $\rho_{g}$, and $\left\|\Delta \mathbf{T}_{f}\right\| \leq \rho_{f}$. For the disturbance, it is assumed that $\mathbf{T}_{L} \in L_{2}[0, T]$, for all $T \in[0, \infty)$, and $\mathbf{T}_{L}$ is bounded by some positive constant $\rho_{d}:\left\|\mathbf{T}_{L}\right\| \leq \rho_{d}$. Thus, the lumped uncertainty is assumed to be bounded by a finite value. To guarantee more improved control performance, an elaborate 
nonlinear friction model should be considered. The state equations for (1)-(4) are represented as

$$
\begin{gathered}
\dot{\mathbf{x}}_{10}=\mathbf{x}_{20} \\
\dot{\mathbf{x}}_{20}=\mathbf{f}_{n}\left(\mathbf{x}_{10}, \mathbf{x}_{20}\right)-\mathbf{g}_{n}\left(\mathbf{x}_{10}\right) \mathbf{T}_{f}+\mathbf{g}_{n}\left(\mathbf{x}_{10}\right) \mathbf{T}_{u}+\mathbf{g}_{n}\left(\mathbf{x}_{10}\right) \tau \\
\dot{\mathbf{x}}_{30}=-\mathbf{L}_{m}^{-1} \mathbf{R}_{m} \mathbf{x}_{30}-\mathbf{L}_{m}^{-1} \mathbf{k}_{b} \mathbf{x}_{20}+\mathbf{L}_{m}^{-1} \mathbf{V}
\end{gathered}
$$

where $\mathbf{x}_{10}=\mathbf{q}, \mathbf{x}_{20}=\dot{\mathbf{q}}$, and $\mathbf{x}_{30}=\mathbf{i}$.

The deadzone nonlinearities $\boldsymbol{\tau}$ are shown in Figure 1(a) and their mathematical models are described by

$$
\boldsymbol{\tau}(t)=D(\mathbf{u})= \begin{cases}\mathbf{m}_{r}\left(\mathbf{u}(t)-\mathbf{B}_{r}\right) & \text { for } \mathbf{u}(t) \geq \mathbf{B}_{r} \\ 0 & \text { for } \mathbf{B}_{l}<\mathbf{u}(t)<\mathbf{B}_{r} \\ \mathbf{m}_{l}\left(\mathbf{u}(t)-\mathbf{B}_{l}\right) & \text { for } \mathbf{u}(t) \leq \mathbf{B}_{l},\end{cases}
$$

where $\mathbf{m}_{r}$ and $\mathbf{m}_{l}$ denote the slope of the deadzone and $\mathbf{B}_{r}$ and $\mathbf{B}_{l}$ stand for the deadzone width parameters. In the control problem, the practical assumptions of the deadzone are as follows.

Assumption 1. The deadzone outputs $\boldsymbol{\tau}(t)$ are not available for measurement. Furthermore, the deadzone parameters $\mathbf{m}_{r}$, $\mathbf{m}_{l}, \mathbf{B}_{r}$, and $\mathbf{B}_{l}$ are unknown but their signs are known, $\mathbf{m}_{r}>$ $0, \mathbf{m}_{l}>0, \mathbf{B}_{r} \geq 0$, and $\mathbf{B}_{l} \leq 0$.

Assumption 2. The deadzone slopes are bounded by known constants $\mathbf{m}_{r \text { min }}, \mathbf{m}_{r \text { max }}, \mathbf{m}_{l \text { min }}$, and $\mathbf{m}_{r \text { max }}$ such that $0<$ $\mathbf{m}_{r \text { min }} \leq \mathbf{m}_{r} \leq \mathbf{m}_{r \text { max }}$ and $0<\mathbf{m}_{l \text { min }} \leq \mathbf{m}_{l} \leq \mathbf{m}_{l \max }$.

The deadzone inverse technique is a useful method to compensate for the deadzone effect [13]. Letting $\mathbf{u}_{d}(t)$ be the signal from the controller that does not take into account the deadzone, the following control signal $\mathbf{u}(t)$ is generated according to the certainty equivalence deadzone inverse described in Figure 1(b):

$$
\begin{aligned}
\mathbf{u}(t)=D^{-1}\left(\mathbf{u}_{d}\right)= & \widehat{\mathbf{m}}_{r}^{-1}\left(\mathbf{u}_{d}(t)+\widehat{\mathbf{B}}_{m r}\right) \boldsymbol{\delta} \\
& +\widehat{\mathbf{m}}_{l}^{-1}\left(\mathbf{u}_{d}(t)+\widehat{\mathbf{B}}_{m l}\right)(\mathbf{I}-\boldsymbol{\delta}),
\end{aligned}
$$

where $\widehat{\mathbf{m}}_{r}, \widehat{\mathbf{m}}_{l}, \widehat{\mathbf{B}}_{m r}$, and $\widehat{\mathbf{B}}_{m l}$ are the estimates of $\mathbf{m}_{r}, \mathbf{m}_{l}, \mathbf{m}_{r} \mathbf{B}_{r}$, and $\mathbf{m}_{l} \mathbf{B}_{l}$, respectively, and

$$
\boldsymbol{\delta}= \begin{cases}\mathbf{I} & \text { if } \mathbf{u}_{d}(t) \geq \mathbf{0} \\ \mathbf{0} & \text { if } \mathbf{u}_{d}(t)<\mathbf{0} .\end{cases}
$$

The resulting errors between $\mathbf{u}$ and $\mathbf{u}_{d}$ are given by

$$
\begin{aligned}
\boldsymbol{\tau}(t)-\mathbf{u}_{d}(t)= & \left(\widetilde{\mathbf{B}}_{m r}-\widehat{\mathbf{m}}_{r}^{-1}\left(\mathbf{u}_{d}(t)+\widehat{\mathbf{B}}_{m r} \widetilde{\mathbf{m}}_{r}\right)\right) \boldsymbol{\delta} \\
& +\left(\widetilde{\mathbf{B}}_{m l}-\widehat{\mathbf{m}}_{l}^{-1}\left(\mathbf{u}_{d}(t)+\widehat{\mathbf{B}}_{m l} \widetilde{\mathbf{m}}_{l}\right)\right)(\mathbf{I}-\boldsymbol{\delta})+\boldsymbol{\varepsilon}_{d},
\end{aligned}
$$

where $\boldsymbol{\varepsilon}_{d}(t)$ is known as the bounded function for all $\mathbf{u}(t)$ [13].

The nonlinear friction forces are assumed to be modeled as

$$
\mathbf{T}_{f}=\sigma_{0} \mathbf{z}+\sigma_{1} \dot{\mathbf{z}}+\boldsymbol{\sigma}_{2} \mathbf{v}+\varepsilon_{f}
$$

where $\sigma_{0}>0$ is the stiffness of the elastic bristle, $\sigma_{1}>0$ is the damping coefficients in the presliding range, $\sigma_{2}>$ 0 is the viscous damping coefficients, and $\boldsymbol{\varepsilon}_{f}$ contains the bounded friction modeling errors. The presliding states $\mathbf{z}$ are represented by the following Elastoplastic model [15]:

$$
\dot{\mathbf{z}}=\mathbf{v}-\boldsymbol{\theta} \boldsymbol{\sigma}_{0} \mathbf{h}(\mathbf{v}) \mathbf{z}
$$

where

$$
h_{i}\left(v_{i}\right)=\frac{\left|v_{i}\right|}{F_{\mathrm{ci}}+\left(F_{\mathrm{si}}-F_{\mathrm{ci}}\right) \exp \left(-\left(v_{i} / v_{\mathrm{si}}\right)^{2}\right)}, \quad i=1, \ldots, n,
$$

$F_{\mathrm{ci}}$ is the Coulomb friction, $F_{\mathrm{si}}$ is the stiction level, $v_{i}$ is the relative velocity between two contact surfaces, $v_{\mathrm{si}}$ is the Stribeck velocity, and $\boldsymbol{\theta}$ is the unknown coefficient related to the presliding friction behavior. The function $h_{i}\left(v_{i}\right)$ is positive and depends on many factors such as material properties, lubrication, and temperature. As the state variables $\mathbf{z}$ cannot be measured directly, we use the friction state observers to estimate $\mathbf{z}$ as follows [19]:

$$
\dot{\widehat{\mathbf{z}}}=\mathbf{v}-\widehat{\boldsymbol{\theta}} \widehat{\boldsymbol{\sigma}}_{0} \mathbf{h}(\mathbf{v}) \widehat{\mathbf{z}}
$$

where $\widehat{\mathbf{z}}, \widehat{\boldsymbol{\theta}}$, and $\widehat{\boldsymbol{\sigma}}_{0}$ are the estimations of $\mathbf{z}, \boldsymbol{\theta}$, and $\boldsymbol{\sigma}_{0}$, respectively. The estimations of the friction $\mathbf{T}_{f}$ can be expressed as

$$
\widehat{\mathbf{T}}_{f}=\widehat{\boldsymbol{\sigma}}_{0} \widehat{\mathbf{z}}+\widehat{\boldsymbol{\sigma}}_{1} \dot{\widehat{\mathbf{z}}}+\widehat{\boldsymbol{\sigma}}_{2} \mathbf{v}
$$

where $\widehat{\boldsymbol{\sigma}}_{1}$ and $\widehat{\boldsymbol{\sigma}}_{2}$ are the estimations of $\boldsymbol{\sigma}_{1}$ and $\boldsymbol{\sigma}_{2}$, respectively. From (10), (11), (13), and (14), we have

$$
\begin{gathered}
\dot{\dot{\mathbf{z}}}=-\boldsymbol{\theta} \boldsymbol{\sigma}_{0} \mathbf{h} \widetilde{\mathbf{z}}-\boldsymbol{\theta} \mathbf{h} \widehat{\mathbf{z}} \widetilde{\boldsymbol{\sigma}}_{0}-\widehat{\boldsymbol{\sigma}}_{0} \mathbf{h} \widehat{\mathbf{z}} \widetilde{\boldsymbol{\theta}}, \\
\widetilde{\mathbf{T}}_{f}=\mathbf{T}_{f}-\widehat{\mathbf{T}}_{f} \\
=\left(\boldsymbol{\sigma}_{0}-\boldsymbol{\theta} \boldsymbol{\sigma}_{1} \mathbf{h}\right) \widetilde{\mathbf{z}}+\left(\widehat{\mathbf{z}}-\boldsymbol{\sigma}_{1} \boldsymbol{\theta} \widehat{\mathbf{z}} \mathbf{h}\right) \widetilde{\boldsymbol{\sigma}}_{0} \\
+(\mathbf{v}-\widehat{\boldsymbol{\theta}} \widehat{\mathbf{z}} \mathbf{h}) \widetilde{\boldsymbol{\sigma}}_{1}+\mathbf{v} \widetilde{\boldsymbol{\sigma}}_{2}-\boldsymbol{\sigma}_{1} \widehat{\boldsymbol{\sigma}}_{1} \widehat{\mathbf{z}} \mathbf{h} \tilde{\boldsymbol{\theta}}+\boldsymbol{\varepsilon}_{f},
\end{gathered}
$$

where $\widetilde{\mathbf{z}}=\mathbf{z}-\widehat{\mathbf{z}}, \widetilde{\boldsymbol{\sigma}}_{(\cdot)}=\boldsymbol{\sigma}_{(\cdot)}-\widehat{\boldsymbol{\sigma}}_{(\cdot)}$, and $\widetilde{\boldsymbol{\theta}}=\boldsymbol{\theta}-\widehat{\boldsymbol{\theta}}$

We can transform the above state model into the following form:

$$
\begin{gathered}
\dot{\mathbf{x}}_{1}=\mathbf{x}_{2} \\
\dot{\mathbf{x}}_{2}=\mathbf{x}_{3}+\mathbf{f}_{2}\left(\underline{\mathbf{x}}_{2}\right)-\mathbf{g}_{n} \mathbf{T}_{f} \\
+\mathbf{g}_{n}\left[\widetilde{\mathbf{B}}_{m r}-\widehat{\mathbf{m}}_{r}^{-1}\left(\mathbf{g}_{n}^{-1} \mathbf{x}_{3}+\widehat{\mathbf{B}}_{m r} \widetilde{\mathbf{m}}_{r}\right)\right] \boldsymbol{\delta} \\
+\mathbf{g}_{n}\left[\widetilde{\mathbf{B}}_{m l}-\widehat{\mathbf{m}}_{l}^{-1}\left(\mathbf{g}_{n}^{-1} \mathbf{x}_{3}+\widehat{\mathbf{B}}_{m l} \widetilde{\mathbf{m}}_{l}\right)\right](\mathbf{I}-\boldsymbol{\delta})+\mathbf{T}_{d} \\
\dot{\mathbf{x}}_{3}=\mathbf{f}_{3}\left(\underline{\mathbf{x}}_{3}\right)+\mathbf{b}_{3} \mathbf{u}_{v},
\end{gathered}
$$

where $\underline{\mathbf{x}}_{3}=\left[\begin{array}{lll}\mathbf{x}_{1} & \mathbf{x}_{2} & \mathbf{x}_{3}\end{array}\right]^{T}=\left[\begin{array}{lll}\mathbf{x}_{10} & \mathbf{x}_{20} & \mathbf{g}_{n} \mathbf{n} \mathbf{k}_{t} \mathbf{x}_{30}\end{array}\right]^{T}$, $\mathbf{f}_{2}\left(\underline{\mathbf{x}}_{2}\right)=\mathbf{f}_{n}\left(\mathbf{x}_{10}, \mathbf{x}_{20}\right), \mathbf{T}_{d}=\mathbf{g}_{n}\left(\mathbf{x}_{1}\right) \mathbf{T}_{u}+\mathbf{g}_{n} \boldsymbol{\varepsilon}_{d}, \mathbf{b}_{3}=\mathbf{g}_{n} \mathbf{n} \mathbf{k}_{t} \mathbf{L}_{m}^{-1}$, and $\mathbf{f}_{3}\left(\underline{\mathbf{x}}_{3}\right)=-\mathbf{g}_{n} \mathbf{n} \mathbf{k}_{t} \mathbf{L}_{m}^{-1}\left(\mathbf{R}_{m} \mathbf{x}_{30}+\mathbf{k}_{b} \mathbf{x}_{20}\right), \underline{\mathbf{x}}_{2}=\left[\begin{array}{ll}\mathbf{x}_{1} & \mathbf{x}_{2}\end{array}\right]^{T}$, and $\mathbf{u}_{v}=\mathbf{V}$ 


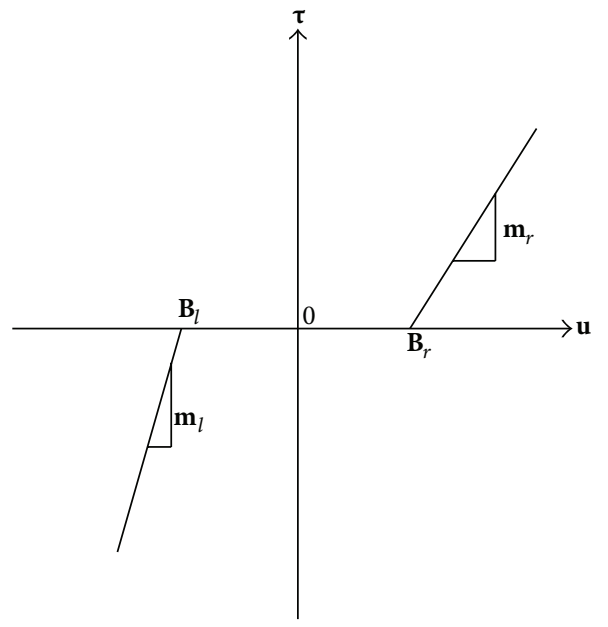

(a) Deadzone

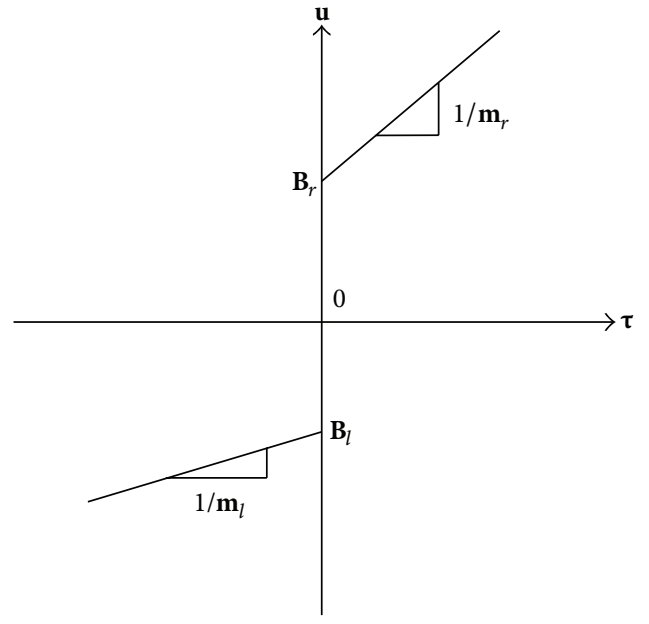

(b) Inverse deadzone

FIgURE 1: Description of deadzone.

2.2. Function Approximation Using a Fuzzy Logic System. The basic configuration of a fuzzy system consists of the fuzzifier, fuzzy rule base, fuzzy inference engine, and defuzzifier. The fuzzy inference engine performs a mapping from an input linguistic vector $x=\left[x_{1}, \ldots, x_{n}\right]^{T} \in R^{n}$ to an output linguistic scalar variable $y \in R$. The fuzzy rule base consists of a collection of fuzzy IF-THEN rules. The $l$ th IF-THEN rules are described by

$$
\begin{gathered}
R^{(l)}: \text { IF } x_{1} \text { is } F_{1}^{l} \text { and } \cdots \text { and } x_{n} \text { is } F_{n}^{l}, \\
\text { then } y \text { is } G^{l}, \quad l=1,2, \ldots, M \text {, }
\end{gathered}
$$

where $F_{i}^{l}, i=1, \ldots, n$, and $G^{l}$ are fuzzy sets characterized by the fuzzy membership functions $\mu_{F_{i}^{l}}\left(x_{i}\right)$ and $\mu_{G^{l}}(y)$, respectively, and $M$ is the number of rules in the fuzzy rule base. The output of a fuzzy system with a center-average defuzzifier, product inference, and singleton fuzzifier is expressed as

$$
y(x)=\frac{\sum_{l=1}^{M} \bar{y}^{l}\left(\prod_{i=1}^{n} \mu_{F_{i}^{l}}\left(x_{i}\right)\right)}{\sum_{l=1}^{M}\left(\prod_{i=1}^{n} \mu_{F_{i}^{l}}\left(x_{i}\right)\right)},
$$

where $\bar{y}^{l}$ is the point at which $\mu_{G^{l}}\left(\bar{y}^{l}\right)=1$ (its maximum value). This equation can be rewritten as

$$
y(x)=W_{o}^{T} X(x),
$$

where $W_{o}^{T}=\left[\bar{y}^{1}, \ldots, \bar{y}^{M}\right]^{T}$ is a vector that groups all the consequence parameters, and $X(x)=\left[X_{1}, \ldots, X_{n}\right]^{T}$ is a set of fuzzy basis functions defined as

$$
X^{l}(x)=\frac{\prod_{i=1}^{n} \mu_{F_{i}^{l}}\left(x_{i}\right)}{\sum_{l=1}^{M}\left(\prod_{i=1}^{n} \mu_{F_{i}^{l}}\left(x_{i}\right)\right)} .
$$

It has been proven that a fuzzy logic system can approximate any nonlinear continuous function to an arbitrary degree accuracy if enough rules are provided [24]. Thus, a fuzzy logic system performs a universal approximation in the sense that, given any real continuous function $f(\cdot): R^{n} \rightarrow R$ on a sufficiently large compact set $\Omega \subset R$ and an arbitrary $\varepsilon_{m}>0$, there exists a fuzzy logic system $y(x)$ in the form of (21) such that

$$
\sup _{x \in \Omega}|f(x)-y(x)| \leq \varepsilon_{m} .
$$

Then the function $f(x)$ can be expressed as

$$
f(x)=W_{o}^{* T} X(x)+\mathcal{\varepsilon}, \quad \forall x \in \Omega \subset R^{n},
$$

where $|\varepsilon| \leq \varepsilon_{m}, \varepsilon^{*}$ is the error of the fuzzy approximation and $W^{*}$ is chosen to be the value of $W$ that minimizes the fuzzy approximation error $\varepsilon$; that is,

$$
W_{o}^{*}=\arg \min _{W_{o} \in R^{M}}\left\{\sup _{x \in \Omega}\left|f(x)-W_{o}^{T} X(x)\right|\right\} .
$$

Since $W_{o}^{*}$ is unknown, it is replaced by $W_{o}$, an estimation of $W_{o}^{*}$. Adaptation laws are required to update the parameter $W_{o}$ and other related fuzzy parameters online to asymptotically minimize the reference tracking error. The optimal fuzzy output function can be rewritten as

$$
W_{o}^{* T} \xi(x)=W_{o}^{T} \xi(x)+\widetilde{W}_{o}^{T} \xi(x),
$$

where $\widetilde{W}_{o}=W_{o}^{*}-W_{o}$.

\section{Design of Controller and Nonsmooth Nonlinear Compensator}

In this section, the adaptive laws and controller are derived via recursive DSC design procedures. The control objective for a robot manipulator system is to determine a state feedback control system such that the system output $\mathbf{x}_{1}$ can track a desired trajectory $\mathbf{y}_{d}$. We add a final assumption to the system. 
Assumption 3. The desired trajectory vectors are continuous and available, and $\left[\mathbf{y}_{d}, \dot{\mathbf{y}}_{d}, \ddot{\mathbf{y}}_{d}\right]^{T} \in \Omega_{d}$ with the known compact set $\Omega_{d}=\left\{\left[\mathbf{y}_{d}, \dot{\mathbf{y}}_{d}, \ddot{\mathbf{y}}_{d}\right]^{T}:\left\|\mathbf{y}_{d}\right\|^{2}+\left\|\dot{\mathbf{y}}_{d}\right\|^{2}+\left\|\ddot{\mathbf{y}}_{d}\right\|^{2} \leq \delta_{y}\right\}$, where $\delta_{y}>0$ is a constant. The state feedback control system is designed step-by-step using a DSC technique as follows.

Step 1. We define the tracking error to be the first error

$$
\mathbf{S}_{1}=\mathbf{x}_{1}-\mathbf{y}_{d}
$$

where time derivative of (27) is

$$
\dot{\mathrm{S}}_{1}=\mathbf{x}_{2}-\dot{\mathbf{y}}_{d}
$$

We define the following Lyapunov function:

$$
V_{1}=\frac{1}{2} \mathbf{S}_{1}^{T} \mathbf{S}_{1}
$$

and its time derivative is given as

$$
\dot{V}_{1}=\mathbf{S}_{1}^{T} \dot{\mathbf{S}}_{1}=\mathbf{S}_{1}^{T}\left(\mathbf{x}_{2}-\dot{\mathbf{y}}_{d}\right) .
$$

We choose a virtual control law to be

$$
\boldsymbol{\alpha}_{1}=-\mathrm{c}_{1} \mathbf{S}_{1}+\dot{\mathbf{y}}_{d}
$$

where $\mathbf{c}_{1}>\mathbf{I}$ is a design constant. We introduce the filtering virtual control $\xi_{2}$ and let $\boldsymbol{\alpha}_{1}$ pass through a first-order filter with a time constant $\varsigma_{2}$ as

$$
\varsigma_{2} \dot{\xi}_{2}+\xi_{2}=\alpha_{1}, \quad \xi_{2}(0)=\alpha_{1}(0) .
$$

Setting $\lambda_{2}=\xi_{2}-\alpha_{1}$, from (25), it follows that

$$
\dot{\xi}_{2}=-\frac{\lambda_{2}}{\varsigma_{2}} .
$$

By using the definition of $\mathbf{x}_{2}=\mathbf{S}_{2}+\boldsymbol{\xi}_{2}$, (30) becomes

$$
\dot{V}_{1} \leq-\mathrm{c}_{1} \mathbf{S}_{1}^{T} \mathbf{S}_{1}+\mathbf{S}_{1}^{T} \mathbf{S}_{2}+\mathbf{S}_{1}^{T} \boldsymbol{\lambda}_{2} .
$$

From (24) and (26), it follows that

$$
\begin{aligned}
\dot{\lambda}_{2} & =\dot{\boldsymbol{\xi}}_{2}-\dot{\boldsymbol{\alpha}}_{1} \\
& =-\frac{\lambda_{2}}{\varsigma_{2}}+\mathrm{c}_{1} \dot{\mathbf{S}}_{1}-\ddot{\mathbf{y}}_{d} \\
& \leq-\frac{\lambda_{2}}{\varsigma_{2}}+\psi_{2}\left(\mathbf{S}_{1}, \mathbf{S}_{2}, \lambda_{2}, \mathbf{y}_{d}, \dot{\mathbf{y}}_{d}, \ddot{\mathbf{y}}_{d}\right), \\
\| \dot{\lambda}_{2} & +\frac{\lambda_{2}}{\varsigma_{2}} \| \leq \psi_{2}\left(\mathbf{S}_{1}, \mathbf{S}_{2}, \lambda_{2}, \mathbf{y}_{d}, \dot{\mathbf{y}}_{d}, \ddot{\mathbf{y}}_{d}\right),
\end{aligned}
$$

where $\psi_{2}\left(\mathbf{S}_{1}, \mathbf{S}_{2}, \lambda_{2}, \mathbf{y}_{d}, \dot{\mathbf{y}}_{d}, \ddot{\mathbf{y}}_{d}\right)$ is a continuous function. From (35) and (36), we have

$$
\begin{aligned}
\lambda_{2}^{T} \dot{\lambda}_{2} & \leq-\frac{\left\|\lambda_{2}\right\|^{2}}{\varsigma_{2}}+\left\|\lambda_{2}\right\| \psi_{2} \\
& \leq-\frac{\left\|\lambda_{2}\right\|^{2}}{\varsigma_{2}}+\left\|\lambda_{2}\right\|^{2}+\frac{1}{4}\left\|\psi_{2}\right\|^{2} .
\end{aligned}
$$

Step 2. We consider the following expression:

$$
\begin{aligned}
\dot{\mathbf{x}}_{2}= & \mathbf{x}_{3}+\mathbf{W}_{o 2}^{* T} \mathbf{X}_{2}\left(\underline{\mathbf{x}}_{2}\right)+\boldsymbol{\varepsilon}_{2}^{*}-\mathbf{g}_{n} \mathbf{T}_{f} \\
& +\mathbf{g}_{n}\left[\widetilde{\mathbf{B}}_{m r}-\widehat{\mathbf{m}}_{r}^{-1}\left(\mathbf{g}_{n}^{-1} \mathbf{x}_{3}+\widehat{\mathbf{B}}_{m r} \widetilde{\mathbf{m}}_{r}\right)\right] \boldsymbol{\delta} \\
& +\mathbf{g}_{n}\left[\widetilde{\mathbf{B}}_{m l}-\widehat{\mathbf{m}}_{l}^{-1}\left(\mathbf{g}_{n}^{-1} \mathbf{x}_{3}+\widehat{\mathbf{B}}_{m l} \widetilde{\mathbf{m}}_{l}\right)\right](\mathbf{I}-\boldsymbol{\delta})+\mathbf{T}_{d} .
\end{aligned}
$$

By defining $\mathbf{S}_{2}=\mathbf{x}_{2}-\boldsymbol{\xi}_{2}$, the time derivative of $\mathbf{S}_{2}$ is given by

$$
\begin{aligned}
\dot{\mathbf{S}}_{2}= & \mathbf{x}_{3}+\mathbf{W}_{o 2}^{T} \mathbf{X}_{2}\left(\underline{\mathbf{x}}_{2}\right)+\widetilde{\mathbf{W}}_{o 2}^{T} \mathbf{X}_{2}\left(\underline{\mathbf{x}}_{2}\right)+\boldsymbol{\varepsilon}_{2}^{*}-\mathbf{g}_{n} \widehat{\mathbf{T}}_{f} \\
& -\mathbf{g}_{n} \widetilde{\mathbf{T}}_{f}+\mathbf{g}_{n}\left[\widetilde{\mathbf{B}}_{m r}-\widehat{\mathbf{m}}_{r}^{-1}\left(\mathbf{g}_{n}^{-1} \mathbf{x}_{3}+\widehat{\mathbf{B}}_{m r} \widetilde{\mathbf{m}}_{r}\right)\right] \boldsymbol{\delta} \\
& +\mathbf{g}_{n}\left[\widetilde{\mathbf{B}}_{m l}-\widehat{\mathbf{m}}_{l}^{-1}\left(\mathbf{g}_{n}^{-1} \mathbf{x}_{3}+\widehat{\mathbf{B}}_{m l} \widetilde{\mathbf{m}}_{l}\right)\right](\mathbf{I}-\boldsymbol{\delta})+\mathbf{T}_{d}-\dot{\boldsymbol{\xi}}_{2} .
\end{aligned}
$$

Next, we define the Lyapunov function candidate:

$$
V_{2}=\frac{1}{2} \mathbf{S}_{2}^{T} \mathbf{S}_{2}
$$

By differentiating (40) with respect to time, we obtain the following equation:

$$
\begin{aligned}
\dot{V}_{2}= & \mathbf{S}_{2}^{T} \dot{\mathbf{S}}_{2} \\
\leq & \mathbf{S}_{2}^{T}\left(\mathbf{x}_{3}+\mathbf{W}_{o 2}^{T} \mathbf{X}_{2}\left(\underline{\mathbf{x}}_{2}\right)-\mathbf{g}_{n} \widehat{\mathbf{T}}_{f}-\dot{\boldsymbol{\xi}}_{2}+\widehat{\boldsymbol{\rho}}_{2} \tanh \left(\boldsymbol{\kappa}_{2}^{-1} \mathbf{S}_{2} \widehat{\boldsymbol{\rho}}_{2}\right)\right) \\
& +\mathbf{S}_{2}^{T} \widetilde{\mathbf{W}}_{o 2}^{T} \mathbf{X}_{2}\left(\underline{\mathbf{x}}_{2}\right)-\mathbf{S}_{2}^{T} \mathbf{g}_{n} \widetilde{\mathbf{T}}_{f}-\left\|\mathbf{S}_{2}\right\| \widetilde{\boldsymbol{\rho}}_{2} \\
& +\mathbf{S}_{2}^{T} \mathbf{g}_{n}\left[\widetilde{\mathbf{B}}_{m r}-\widehat{\mathbf{m}}_{r}^{-1}\left(\mathbf{g}_{n}^{-1} \mathbf{x}_{3}+\widehat{\mathbf{B}}_{m r} \widetilde{\mathbf{m}}_{r}\right)\right] \boldsymbol{\delta} \\
& +\mathbf{S}_{2}^{T} \mathbf{g}_{n}\left[\widetilde{\mathbf{B}}_{m l}-\widehat{\mathbf{m}}_{l}^{-1}\left(\mathbf{g}_{n}^{-1} \mathbf{x}_{3}+\widehat{\mathbf{B}}_{m l} \widetilde{\mathbf{m}}_{l}\right)\right](\mathbf{I}-\boldsymbol{\delta})+\kappa_{2}^{\prime},
\end{aligned}
$$

where $\left\|\boldsymbol{\varepsilon}_{i 2}+\mathbf{T}_{d}\right\| \leq\left\|\boldsymbol{\rho}_{2}\right\|, 0 \leq\left|S_{2 i} \rho_{2 i}\right|-S_{2 i} \widehat{\rho}_{2 i} \tanh \left(S_{2 i} \widehat{\rho}_{2 i} / \kappa_{2 i}\right) \leq$ $0.2785 \kappa_{2 i}=\kappa_{2 i}^{\prime}, i=1, \ldots, n, \kappa_{2 i}$ is a design constant, and where $\widetilde{\boldsymbol{\rho}}_{2}=\widehat{\boldsymbol{\rho}}_{2}-\boldsymbol{\rho}_{2}$. We specify a virtual control $\boldsymbol{\alpha}_{2}$ to be as follows:

$$
\begin{aligned}
\boldsymbol{\alpha}_{2}= & -\mathbf{c}_{2} \mathbf{S}_{2}-\mathbf{S}_{1}-\mathbf{W}_{o 2}^{T} \mathbf{X}_{2}\left(\underline{\mathbf{x}}_{2}\right)+\dot{\boldsymbol{\xi}}_{2}+\mathbf{g}_{n} \widehat{\mathbf{T}}_{f} \\
& -\mathbf{S}_{2} \widehat{\boldsymbol{\rho}}_{2} \tanh \left(\boldsymbol{\kappa}_{2}^{-1} \mathbf{S}_{2} \widehat{\boldsymbol{\rho}}_{2}\right)
\end{aligned}
$$

where $\boldsymbol{c}_{2}>\mathbf{I}$ is a design constant and $\hat{\boldsymbol{\rho}}_{2}$ is the estimation of $\boldsymbol{\rho}_{2}$. We introduce a new filtering virtual control $\boldsymbol{\xi}_{3}$ and let $\boldsymbol{\alpha}_{2}$ pass through a first-order filter with a time constant $\varsigma_{3}$ as

$$
\varsigma_{3} \dot{\xi}_{3}+\xi_{3}=\alpha_{2}, \quad \xi_{3}(0)=\alpha_{2}(0) .
$$

By setting $\lambda_{3}=\xi_{3}-\alpha_{2}$, from (36), it follows that

$$
\dot{\xi}_{3}=-\frac{\lambda_{3}}{\varsigma_{3}} .
$$

We define $\mathbf{S}_{3}=\mathbf{x}_{3}-\boldsymbol{\xi}_{3}$. It then follows that

$$
\begin{aligned}
\mathbf{x}_{3}= & \mathbf{S}_{3}+\boldsymbol{\lambda}_{3}-\mathbf{c}_{2} \mathbf{S}_{2}-\mathbf{S}_{1}-\mathbf{W}_{o 2}^{T} \mathbf{X}_{2}\left(\underline{\mathbf{x}}_{2}\right)+\dot{\boldsymbol{\xi}}_{2} \\
& +\mathbf{g}_{n} \widehat{\mathbf{T}}_{f}-\widehat{\boldsymbol{\rho}}_{2} \tanh \left(\boldsymbol{\kappa}_{2}^{-1} \mathbf{S}_{2} \widehat{\boldsymbol{\rho}}_{2}\right) .
\end{aligned}
$$


Substituting (45) into (41), we obtain the following expression:

$$
\begin{aligned}
\dot{V}_{2} \leq & -\mathbf{c}_{2} \mathbf{S}_{2}^{T} \mathbf{S}_{2}-\mathbf{S}_{2}^{T} \mathbf{S}_{1}+\mathbf{S}_{2}^{T} \mathbf{S}_{3}+\mathbf{S}_{2}^{T} \boldsymbol{\lambda}_{3} \\
& +\mathbf{S}_{2}^{T} \widetilde{\mathbf{W}}_{o 2}^{T} \mathbf{X}_{2}\left(\underline{\mathbf{x}}_{2}\right)-\mathbf{S}_{2}^{T} \mathbf{g}_{n} \widetilde{\mathbf{T}}_{f}-\left\|\mathbf{S}_{2}\right\| \widetilde{\boldsymbol{\rho}}_{2} \\
& +\mathbf{S}_{2}^{T} \mathbf{g}_{n}\left[\widetilde{\mathbf{B}}_{m r}-\widehat{\mathbf{m}}_{r}^{-1}\left(\mathbf{g}_{n}^{-1} \mathbf{x}_{3}+\widehat{\mathbf{B}}_{m r} \widetilde{\mathbf{m}}_{r}\right)\right] \boldsymbol{\delta} \\
& +\mathbf{S}_{2}^{T} \mathbf{g}_{n}\left[\widetilde{\mathbf{B}}_{m l}-\widehat{\mathbf{m}}_{l}^{-1}\left(\mathbf{g}_{n}^{-1} \mathbf{x}_{3}+\widehat{\mathbf{B}}_{m l} \widetilde{\mathbf{m}}_{l}\right)\right](\mathbf{I}-\boldsymbol{\delta})+\boldsymbol{\kappa}_{2}^{\prime}
\end{aligned}
$$

From (36), it follows that

$$
\begin{aligned}
\dot{\lambda}_{3}= & \dot{\boldsymbol{\xi}}_{3}-\dot{\boldsymbol{\alpha}}_{2} \\
= & -\frac{\lambda_{3}}{\varsigma_{3}}+\mathbf{c}_{2} \dot{\mathbf{S}}_{2}+\dot{\mathbf{W}}_{o 2}^{T} \mathbf{X}_{2}\left(\underline{\mathbf{x}}_{2}\right)+\mathbf{W}_{o 2}^{T} \frac{\partial \mathbf{X}_{2}\left(\underline{\mathbf{x}}_{2}\right)}{\partial \underline{\mathbf{x}}_{2}} \dot{\mathbf{x}}_{2} \\
& -\dot{\widehat{\mathbf{T}}}_{f}-\ddot{\boldsymbol{\xi}}_{2}+\frac{d\left(\widehat{\boldsymbol{\rho}}_{2} \tanh \left(\boldsymbol{\kappa}_{2}^{-1} \mathbf{S}_{2} \widehat{\boldsymbol{\rho}}_{2}\right)\right)}{d \mathbf{S}_{2}} \dot{\mathbf{S}}_{2} \\
\leq & -\frac{\lambda_{3}}{\varsigma_{3}}+\psi_{3}\left(\mathbf{S}_{1}, \mathbf{S}_{2}, \mathbf{S}_{3}, \lambda_{2}, \lambda_{3}, \mathbf{W}_{o 2}, \widehat{\mathbf{T}}_{f}, \mathbf{y}_{d}, \dot{\mathbf{y}}_{d}, \ddot{\mathbf{y}}_{d}\right), \\
\| \dot{\boldsymbol{\lambda}}_{3}+ & \frac{\lambda_{i 3}}{\varsigma_{3}} \| \leq \psi_{3}\left(\mathbf{S}_{1}, \mathbf{S}_{2}, \mathbf{S}_{3}, \lambda_{2}, \lambda_{3}, \mathbf{W}_{o 2}, \widehat{\mathbf{T}}_{f}, \mathbf{y}_{d}, \dot{\mathbf{y}}_{d}, \ddot{\mathbf{y}}_{d}\right),
\end{aligned}
$$

where $\psi_{3}(\cdot)$ is a continuous function. From (47), we obtain the following inequality:

$$
\begin{aligned}
\lambda_{3}^{T} \dot{\lambda}_{3} & \leq-\frac{\left\|\lambda_{3}\right\|^{2}}{\varsigma_{3}}+\left\|\lambda_{3}\right\| \psi_{3} \\
& \leq-\frac{\left\|\lambda_{3}\right\|^{2}}{\varsigma_{3}}+\left\|\lambda_{3}\right\|^{2}+\frac{1}{4}\left\|\psi_{3}\right\|^{2} .
\end{aligned}
$$

Step 3. The final control law is derived in this step. Consider

$$
\dot{\mathbf{x}}_{3}=\mathbf{b}_{3} \mathbf{u}_{v}+\mathbf{W}_{o 3}^{* T} \mathbf{X}_{3}\left(\underline{\mathbf{x}}_{3}\right)+\boldsymbol{\varepsilon}_{3}^{*} .
$$

From the third error surface $S_{3}=\mathbf{x}_{3}-\boldsymbol{\xi}_{3}$, it follows that

$$
\begin{aligned}
\dot{\mathbf{S}}_{3} & =\dot{\mathbf{x}}_{3}-\dot{\boldsymbol{\xi}}_{3} \\
& =\mathbf{b}_{3} \mathbf{u}_{v}+\mathbf{W}_{o 3}^{* T} \mathbf{X}_{3}\left(\underline{\mathbf{x}}_{3}\right)+\boldsymbol{\varepsilon}_{3}^{*}-\dot{\boldsymbol{\xi}}_{3} \\
& \leq \mathbf{b}_{3} \mathbf{u}_{v}+\mathbf{W}_{o 3}^{T} \mathbf{X}_{3}\left(\underline{\mathbf{x}}_{3}\right)-\dot{\boldsymbol{\xi}}_{3}+\widetilde{\mathbf{W}}_{o 3}^{T} \mathbf{X}_{3}\left(\underline{\mathbf{x}}_{3}\right)+\widehat{\boldsymbol{\rho}}_{3}-\widetilde{\boldsymbol{\rho}}_{3},
\end{aligned}
$$

where $\left\|\varepsilon_{3}^{*}\right\| \leq\left\|\rho_{3}\right\|, \rho_{3}$ is a positive constant, and $\widehat{\rho}_{3}$ is an estimation of $\rho_{3}$.

The adaptive strict feedback dynamic surface control is modified to enforce robustness by adding a sliding mode control. The modification starts by defining the following sliding surface in terms of the error coordinates:

$$
\chi=\gamma_{1} S_{1}+\gamma_{2} S_{2}+S_{3}
$$

We define the following Lyapunov function candidate:

$$
\begin{aligned}
V= & \sum_{k=1}^{2} V_{k}+\frac{1}{2} \boldsymbol{\chi}^{T} \boldsymbol{\chi}+\frac{1}{2} \sum_{k=1}^{2} \boldsymbol{\lambda}_{k+1}^{T} \boldsymbol{\lambda}_{k+1} \\
& +\sum_{k=2}^{3} \frac{1}{2 \eta_{w k}} \widetilde{\mathbf{W}}_{o k}^{T} \widetilde{\mathbf{W}}_{o k}+\sum_{k=2}^{3} \frac{1}{2 \eta_{\rho k}} \widetilde{\boldsymbol{\rho}}_{k}^{T} \widetilde{\boldsymbol{\rho}}_{k} \\
& +\frac{1}{2} \mathbf{g}_{n} \widetilde{\mathbf{z}}^{T} \widetilde{\mathbf{z}}+\frac{1}{2 \eta_{0}} \mathbf{g}_{n} \widetilde{\boldsymbol{\sigma}}_{0}^{T} \widetilde{\boldsymbol{\sigma}}_{0}+\frac{1}{2 \eta_{1}} \mathbf{g}_{n} \widetilde{\boldsymbol{\sigma}}_{1}^{T} \widetilde{\boldsymbol{\sigma}}_{1} \\
& +\frac{1}{2 \eta_{2}} \mathbf{g}_{n} \widetilde{\boldsymbol{\sigma}}_{2}^{T} \widetilde{\boldsymbol{\sigma}}_{2}+\frac{1}{2 \eta_{\theta}} \mathbf{g}_{n} \boldsymbol{\sigma}_{1} \widetilde{\boldsymbol{\theta}}^{T} \widetilde{\boldsymbol{\theta}} \\
& +\frac{1}{2 \eta_{m r}} \mathbf{g}_{n} \widetilde{\mathbf{m}}_{r}^{T} \widetilde{\mathbf{m}}_{r}+\frac{1}{2 \eta_{m l}} \mathbf{g}_{n} \widetilde{\mathbf{m}}_{l}^{T} \widetilde{\mathbf{m}}_{l} \\
& +\frac{1}{2 \eta_{b r}} \mathbf{g}_{n} \widetilde{\mathbf{B}}_{m r}^{T} \widetilde{\mathbf{B}}_{m r}+\frac{1}{2 \eta_{b l}} \mathbf{g}_{n} \widetilde{\mathbf{B}}_{m l}^{T} \widetilde{\mathbf{B}}_{m l},
\end{aligned}
$$

where $\eta_{i i}$ are positive constants. The time derivative of $V$ is calculated as

$$
\begin{aligned}
& \dot{V}=\sum_{k=1}^{2} \dot{V}_{k}+\chi^{T} \dot{\chi}+\sum_{k=1}^{2} \boldsymbol{\lambda}_{k+1}^{T} \dot{\lambda}_{k+1} \\
& +\sum_{k=2}^{3} \frac{1}{\eta_{w k}} \widetilde{\mathbf{W}}_{o k}^{T} \dot{\widetilde{\mathbf{W}}}_{o k}+\sum_{k=2}^{3} \frac{1}{\eta_{\rho k}} \widetilde{\boldsymbol{\rho}}_{k}^{T} \dot{\tilde{\boldsymbol{\rho}}}_{k} \\
& +\mathbf{g}_{n} \widetilde{\mathbf{z}}^{T} \dot{\overrightarrow{\mathbf{z}}}+\frac{1}{\eta_{0}} \mathbf{g}_{n} \widetilde{\boldsymbol{\sigma}}_{0}^{T} \dot{\tilde{\boldsymbol{\sigma}}}_{0}+\frac{1}{\eta_{1}} \mathbf{g}_{n} \widetilde{\boldsymbol{\sigma}}_{1}^{T} \dot{\tilde{\boldsymbol{\sigma}}}_{1} \\
& +\frac{1}{\eta_{2}} \mathbf{g}_{n} \widetilde{\boldsymbol{\sigma}}_{2}^{T} \dot{\tilde{\boldsymbol{\sigma}}}_{2}+\frac{1}{\eta_{\theta}} \mathbf{g}_{n} \widehat{\boldsymbol{\sigma}}_{1} \widetilde{\boldsymbol{\theta}}^{T} \dot{\tilde{\boldsymbol{\theta}}} \\
& +\frac{1}{\eta_{m r}} \mathbf{g}_{n} \widetilde{\mathbf{m}}_{r}^{T} \dot{\overline{\mathbf{m}}}_{r}+\frac{1}{\eta_{m l}} \mathbf{g}_{n} \widetilde{\mathbf{m}}_{l}^{T} \dot{\overline{\mathbf{m}}}_{l} \\
& +\frac{1}{\eta_{b r}} \mathbf{g}_{n} \widetilde{\mathbf{B}}_{m r}^{T} \dot{\widetilde{\mathbf{B}}}_{m r}+\frac{1}{\eta_{b l}} \mathbf{g}_{n} \widetilde{\mathbf{B}}_{m l}^{T} \dot{\widetilde{\mathbf{B}}}_{m l} \\
& \leq-\sum_{k=1}^{2} \mathbf{c}_{k} \mathbf{S}_{k}^{T} \mathbf{S}_{k}+\mathbf{S}_{2}^{T} \mathbf{S}_{3}+\sum_{k=1}^{2} \mathbf{S}_{k}^{T} \boldsymbol{\lambda}_{k+1} \\
& +\mathbf{S}_{2}^{T} \widetilde{\mathbf{W}}_{o 2}^{T} \mathbf{X}_{2}\left(\underline{\mathbf{x}}_{2}\right)-\left\|\mathbf{S}_{2}\right\| \widetilde{\boldsymbol{\rho}}_{2}-\mathbf{S}_{2}^{T} \mathbf{g}_{n} \widetilde{\mathbf{T}}_{f} \\
& +\mathbf{S}_{2}^{T} \mathbf{g}_{n}\left[\widetilde{\mathbf{B}}_{m r}-\widehat{\mathbf{m}}_{r}^{-1}\left(\mathbf{g}_{n}^{-1} \mathbf{x}_{3}+\widehat{\mathbf{B}}_{m r} \widetilde{\mathbf{m}}_{r}\right)\right] \boldsymbol{\delta} \\
& +\mathbf{S}_{2}^{T} \mathbf{g}_{n}\left[\widetilde{\mathbf{B}}_{m l}-\widehat{\mathbf{m}}_{l}^{-1}\left(\mathbf{g}_{n}^{-1} \mathbf{x}_{3}+\widehat{\mathbf{B}}_{m l} \widetilde{\mathbf{m}}_{l}\right)\right](\mathbf{I}-\boldsymbol{\delta})+\boldsymbol{\kappa}_{2}^{\prime} \\
& +\chi^{T} \dot{\chi}+\sum_{k=1}^{2} \lambda_{k+1}^{T} \dot{\boldsymbol{\lambda}}_{k+1}+\sum_{k=2}^{3} \frac{1}{\eta_{w k}} \widetilde{\mathbf{W}}_{o k}^{T} \dot{\widetilde{\mathbf{W}}}_{o k} \\
& +\sum_{k=2}^{3} \frac{1}{\eta_{\rho k}} \widetilde{\boldsymbol{\rho}}_{k}^{T} \dot{\tilde{\boldsymbol{\rho}}}_{k}+\mathbf{g}_{n} \widetilde{\mathbf{z}}^{T} \dot{\overrightarrow{\mathbf{z}}}+\frac{1}{\eta_{0}} \mathbf{g}_{n} \widetilde{\boldsymbol{\sigma}}_{0}^{T} \dot{\widetilde{\boldsymbol{\sigma}}}_{0} \\
& +\frac{1}{\eta_{1}} \mathbf{g}_{n} \tilde{\boldsymbol{\sigma}}_{1}^{T} \dot{\tilde{\boldsymbol{\sigma}}}_{1}+\frac{1}{\eta_{2}} \mathbf{g}_{n} \tilde{\boldsymbol{\sigma}}_{2}^{T} \dot{\boldsymbol{\sigma}}_{2}+\frac{1}{\eta_{\theta}} \mathbf{g}_{n} \widehat{\boldsymbol{\sigma}}_{1} \widetilde{\boldsymbol{\theta}}^{T} \dot{\tilde{\boldsymbol{\theta}}}
\end{aligned}
$$




$$
\begin{aligned}
& +\frac{1}{\eta_{m r}} \mathbf{g}_{n} \widetilde{\mathbf{m}}_{r}^{T} \dot{\mathbf{\mathbf { m }}}_{r}+\frac{1}{\eta_{m l}} \mathbf{g}_{n} \widetilde{\mathbf{m}}_{l}^{T} \dot{\mathbf{\mathbf { m }}}_{l} \\
& +\frac{1}{\eta_{b r}} \mathbf{g}_{n} \widetilde{\mathbf{B}}_{m r}^{T} \dot{\tilde{\mathbf{B}}}_{m r}+\frac{1}{\eta_{b l}} \mathbf{g}_{n} \widetilde{\mathbf{B}}_{m l}^{T} \dot{\tilde{\mathbf{B}}}_{m l} .
\end{aligned}
$$

From (51), we obtain the following relation:

$$
\mathbf{S}_{3}=-\gamma_{1} \mathbf{S}_{1}-\gamma_{2} \mathbf{S}_{2}+\chi .
$$

By considering the previous results and (54), we obtain the following result:

$$
\begin{aligned}
& \dot{V} \leq-\sum_{k=1}^{2} \mathbf{c}_{k} \mathbf{S}_{k}^{T} \mathbf{S}_{k}+\mathbf{S}_{2}^{T}\left(-\boldsymbol{\gamma}_{1} \mathbf{S}_{1}-\boldsymbol{\gamma}_{2} \mathbf{S}_{2}\right) \\
& +\sum_{k=1}^{2}\left\|\mathbf{S}_{k}\right\|\left\|\lambda_{k+1}\right\|+\mathbf{S}_{2}^{T} \widetilde{\mathbf{W}}_{o 2}^{T} \mathbf{X}_{2}\left(\underline{\mathbf{x}}_{2}\right)-\left\|\mathbf{S}_{2}\right\| \widetilde{\boldsymbol{\rho}}_{2}-\mathbf{S}_{2}^{T} \mathbf{g}_{n} \widetilde{\mathbf{T}}_{f} \\
& +\mathbf{S}_{2}^{T} \mathbf{g}_{n}\left[\widetilde{\mathbf{B}}_{m r}-\widehat{\mathbf{m}}_{r}^{-1}\left(\mathbf{g}_{n}^{-1} \mathbf{x}_{3}+\widehat{\mathbf{B}}_{m r} \widetilde{\mathbf{m}}_{r}\right)\right] \boldsymbol{\delta} \\
& +\mathbf{S}_{2}^{T} \mathbf{g}_{n}\left[\widetilde{\mathbf{B}}_{m l}-\widehat{\mathbf{m}}_{l}^{-1}\left(\mathbf{g}_{n}^{-1} \mathbf{x}_{3}+\widehat{\mathbf{B}}_{m l} \widetilde{\mathbf{m}}_{l}\right)\right](\mathbf{I}-\boldsymbol{\delta})+\boldsymbol{\kappa}_{2}^{\prime} \\
& +\chi^{T}\left[\mathbf{S}_{2}^{T}+\gamma_{1}\left(\mathbf{S}_{2}+\boldsymbol{\xi}_{2}-\dot{y}_{d}\right)\right. \\
& +\gamma_{2}\left(\mathbf{S}_{3}+\lambda_{3}-\mathrm{c}_{2} \mathrm{~S}_{2}-\mathrm{S}_{1}\right) \\
& \left.+\mathbf{b}_{3} \mathbf{u}_{v}+\mathbf{W}_{o 3}^{T} \mathbf{X}_{3}\left(\underline{\mathbf{x}}_{3}\right)-\dot{\boldsymbol{\xi}}_{3}+\widehat{\boldsymbol{\rho}}_{3}\right] \\
& +\chi^{T}\left[\boldsymbol{\gamma}_{2} \widetilde{\mathbf{W}}_{o 2}^{T} \mathbf{X}_{2}\left(\underline{\mathbf{x}}_{2}\right)-\mathbf{g}_{n} \boldsymbol{\gamma}_{2} \widetilde{\mathbf{T}}_{f}+\mathbf{g}_{n} \boldsymbol{\gamma}_{2} \widetilde{\mathbf{B}}_{m r} \boldsymbol{\delta}\right. \\
& -\mathbf{g}_{n} \gamma_{2} \widehat{\mathbf{m}}_{r}^{-1} \widehat{\mathbf{B}}_{m r} \widetilde{\mathbf{m}}_{r} \boldsymbol{\delta}+\mathbf{g}_{n} \gamma_{2} \widetilde{\mathbf{B}}_{m l}(\mathbf{I}-\boldsymbol{\delta}) \\
& \left.-\mathbf{g}_{n} \gamma_{2} \widehat{\mathbf{m}}_{l}^{-1} \widehat{\mathbf{B}}_{m l} \widetilde{\mathbf{m}}_{l}(\mathbf{I}-\boldsymbol{\delta})+\widetilde{\mathbf{W}}_{o 3}^{T} \mathbf{X}_{3}\left(\underline{\mathbf{x}}_{3}\right)-\widetilde{\boldsymbol{\rho}}_{3}+\boldsymbol{\kappa}_{2}^{\prime}\right] \\
& -\sum_{k=1}^{2} \frac{\left\|\lambda_{k+1}\right\|^{2}}{\varsigma_{k+1}}+\sum_{k=1}^{2}\left\|\lambda_{k+1}\right\|^{2}+\frac{1}{4} \sum_{k=1}^{2}\left\|\psi_{k+1}\right\|^{2} \\
& +\sum_{k=2}^{3} \frac{1}{\eta_{w k}} \widetilde{\mathbf{W}}_{o k}^{T} \dot{\overline{\mathbf{W}}}_{o k}+\sum_{k=2}^{3} \frac{1}{\eta_{\rho k}} \widetilde{\boldsymbol{\rho}}_{k}^{T} \dot{\tilde{\boldsymbol{\rho}}}_{k} \\
& +\frac{1}{\eta_{b r}} \mathbf{g}_{n} \widetilde{\mathbf{B}}_{m r}^{T} \dot{\tilde{\mathbf{B}}}_{m r}+\mathbf{g}_{n} \widetilde{\mathbf{z}}^{T} \dot{\mathbf{\mathbf { z }}}+\frac{1}{\eta_{0}} \mathbf{g}_{n} \widetilde{\boldsymbol{\sigma}}_{0}^{T} \dot{\tilde{\boldsymbol{\sigma}}}_{0}+\frac{1}{\eta_{1}} \mathbf{g}_{n} \widetilde{\boldsymbol{\sigma}}_{1}^{T} \dot{\tilde{\boldsymbol{\sigma}}}_{1} \\
& +\frac{1}{\eta_{2}} \mathbf{g}_{n} \widetilde{\boldsymbol{\sigma}}_{2}^{T} \dot{\tilde{\boldsymbol{\sigma}}}_{2}+\frac{1}{\eta_{\theta}} \mathbf{g}_{n} \boldsymbol{\sigma}_{1} \widetilde{\boldsymbol{\theta}}^{T} \dot{\tilde{\boldsymbol{\theta}}}+\frac{1}{\eta_{m r}} \mathbf{g}_{n} \widetilde{\mathbf{m}}_{r}^{T} \dot{\mathbf{m}}_{r} \\
& +\frac{1}{\eta_{m l}} \mathbf{g}_{n} \widetilde{\mathbf{m}}_{l}^{T} \dot{\overrightarrow{\mathbf{m}}}_{l}+\frac{1}{\eta_{b r}} \mathbf{g}_{n} \widetilde{\mathbf{B}}_{m r}^{T} \dot{\overrightarrow{\mathbf{B}}}_{m r}+\frac{1}{\eta_{b l}} \mathbf{g}_{n} \widetilde{\mathbf{B}}_{m l}^{T} \dot{\tilde{\mathbf{B}}}_{m l} .
\end{aligned}
$$

We choose the control input to be

$$
\begin{aligned}
\mathbf{u}_{v}=\mathbf{b}_{3}^{-1}[ & -\mathbf{S}_{2}^{T}-\boldsymbol{\gamma}_{1}\left(\mathbf{S}_{2}+\boldsymbol{\xi}_{2}-\dot{y}_{d}\right) \\
& -\gamma_{2}\left(\mathbf{S}_{3}+\lambda_{3}-\mathbf{c}_{2} \mathbf{S}_{2}-\mathbf{S}_{1}\right) \\
& \left.-\mathbf{W}_{o 3}^{T} \mathbf{X}_{3}\left(\underline{\mathbf{x}}_{3}\right)+\widehat{\boldsymbol{\rho}}_{3}-\dot{\boldsymbol{\xi}}_{3}-\mathbf{K}_{1} \boldsymbol{\chi}-\mathbf{K}_{2} \operatorname{sgn}(\chi)\right],
\end{aligned}
$$

where $\mathbf{K}_{1}>0$ and $\mathbf{K}_{2}>0$ are design constants. By using Young's inequality expressed as $\left\|\mathbf{S}_{k}\right\|\left\|\lambda_{k+1}\right\| \leq\left\|\mathbf{S}_{k}\right\|^{2}+$ $(1 / 4)\left\|\lambda_{k+1}\right\|^{2}$, (56) becomes

$$
\dot{V} \leq-\sum_{k=1}^{2}\left(\mathbf{c}_{k}-1\right) \mathbf{S}_{k}^{T} \mathbf{S}_{k}+\mathbf{S}_{2}^{T}\left(-\gamma_{1} \mathbf{S}_{1}-\gamma_{2} \mathbf{S}_{2}\right)
$$

$$
-\mathbf{K}_{1}\|\chi\|^{2}-\mathbf{K}_{2}\|\chi\|
$$$$
+\widetilde{\mathbf{W}}_{o 3}^{T}\left(\chi^{T} \mathbf{X}_{3}\left(\underline{\mathbf{x}}_{3}\right)-\frac{1}{\eta_{w 3}} \dot{\mathbf{W}}_{o 3}\right)+\tilde{\boldsymbol{\rho}}_{3}^{T}\left(-\chi^{T}+\frac{1}{\eta_{\rho 3}} \dot{\hat{\boldsymbol{\rho}}}_{3}\right)
$$$$
+\mathbf{g}_{n} \widetilde{\mathbf{m}}_{r}^{T}\left(-\left(\mathbf{S}_{2}^{T}+\boldsymbol{\gamma}_{2} \chi^{T}\right) \widehat{\mathbf{m}}_{r}^{-1} \widehat{\mathbf{B}}_{m r} \boldsymbol{\delta}-\frac{1}{\eta_{m r}} \dot{\mathbf{m}}_{r}\right)
$$$$
+\mathbf{g}_{n} \widetilde{\mathbf{m}}_{l}^{T}\left(-\left(\mathbf{S}_{2}^{T}+\boldsymbol{\gamma}_{2} \chi^{T}\right) \widehat{\mathbf{m}}_{l}^{-1} \widehat{\mathbf{B}}_{m l}(\mathbf{I}-\boldsymbol{\delta})-\frac{1}{\eta_{m l}} \dot{\mathbf{m}}_{l}\right)
$$$$
+\mathbf{g}_{n} \widetilde{\mathbf{B}}_{m r}^{T}\left(\left(\mathbf{S}_{2}^{T}+\boldsymbol{\gamma}_{2} \boldsymbol{\chi}^{T}\right) \boldsymbol{\delta}-\frac{1}{\eta_{b r}} \dot{\hat{\mathbf{B}}}_{m r}\right)
$$$$
+\mathbf{g}_{n} \widetilde{\mathbf{B}}_{m l}^{T}\left(\left(\mathbf{S}_{2}^{T}+\gamma_{2} \boldsymbol{\chi}^{T}\right)(\mathbf{I}-\boldsymbol{\delta})-\frac{1}{\eta_{b l}} \dot{\widehat{\mathbf{B}}}_{m l}\right)
$$$$
+\widetilde{\mathbf{W}}_{o 2}^{T}\left(\left(\mathbf{S}_{2}^{T}+\gamma_{2} \chi^{T}\right) \mathbf{X}_{2}\left(\underline{\mathbf{x}}_{2}\right)-\frac{1}{\eta_{w 2}} \dot{\mathbf{W}}_{o 2}\right)
$$$$
+\widetilde{\boldsymbol{\rho}}_{2}^{T}\left(-\left\|\mathbf{S}_{2}\right\|+\frac{1}{\eta_{\rho 2}} \dot{\hat{\boldsymbol{\rho}}}_{2}\right)
$$$$
+\mathbf{g}_{n} \tilde{\boldsymbol{\sigma}}_{0}^{T}\left(-\left(\mathbf{S}_{2}^{T}+\gamma_{2} \chi^{T}\right) \widehat{\mathbf{z}}-\frac{1}{\eta_{0}} \dot{\widehat{\boldsymbol{\sigma}}}_{0}\right)
$$$$
+\mathbf{g}_{n} \widetilde{\boldsymbol{\sigma}}_{1}^{T}\left(-\left(\mathbf{S}_{2}^{T}+\gamma_{2} \chi^{T}\right) \dot{\widehat{\mathbf{z}}}-\frac{1}{\eta_{1}} \dot{\widehat{\boldsymbol{\sigma}}}_{1}\right)
$$$$
+\mathbf{g}_{n} \widetilde{\boldsymbol{\sigma}}_{2}^{T}\left(-\left(\mathbf{S}_{2}^{T}+\gamma_{2} \boldsymbol{\chi}^{T}\right) \mathbf{v}-\frac{1}{\eta_{2}} \dot{\hat{\boldsymbol{\sigma}}}_{2}\right)
$$$$
+\mathbf{g}_{n} \boldsymbol{\sigma}_{1} \widetilde{\boldsymbol{\theta}}^{T}\left(-\left(\mathbf{S}_{2}^{T}+\gamma_{2} \chi^{T}\right) \widehat{\boldsymbol{\sigma}}_{1} \widehat{\mathbf{z}} \mathbf{h}-\frac{1}{\eta_{\theta}} \dot{\dot{\boldsymbol{\theta}}}\right)
$$$$
+\frac{5}{4} \sum_{k=1}^{2}\left\|\boldsymbol{\lambda}_{k+1}\right\|^{2}+\frac{1}{4} \sum_{k=1}^{2}\left\|\boldsymbol{\psi}_{k+1}\right\|^{2}+\mathbf{g}_{n} \boldsymbol{\kappa}_{2}^{\prime}
$$$$
-\mathbf{g}_{n}\left(\mathbf{S}_{2}^{T}+\gamma_{2} \chi^{T}\right)\left(\sigma_{0}-\boldsymbol{\theta} \boldsymbol{\sigma}_{1} \mathbf{h}\right) \widetilde{\mathbf{z}}
$$$$
+\mathbf{g}_{n}\left(\mathbf{S}_{2}^{T}+\gamma_{2} \chi^{T}\right) \boldsymbol{\sigma}_{1} \theta \overline{\mathbf{z}} \mathbf{h} \widetilde{\boldsymbol{\sigma}}_{0}-\mathbf{g}_{n}\left(\mathbf{S}_{2}^{T}+\gamma_{2} \chi^{T}\right) \boldsymbol{\varepsilon}_{f}
$$$$
+\mathbf{g}_{n}\left(-\boldsymbol{\theta} \boldsymbol{\sigma}_{0} \mathbf{h} \widetilde{\mathbf{z}}^{T}-\boldsymbol{\theta} \mathbf{h} \widehat{\mathbf{z}} \widetilde{\boldsymbol{\sigma}}_{0}-\widehat{\boldsymbol{\sigma}}_{0} \mathbf{h} \widehat{\mathbf{z}} \widetilde{\boldsymbol{\theta}}\right) \widetilde{\mathbf{z}}
$$

We specify the adaptive laws as follows:

$$
\begin{gathered}
\dot{\mathbf{W}}_{o 2}=\operatorname{Proj}_{w o 2}\left[\eta_{w 2}\left(\mathbf{s}_{2}^{T}+\gamma_{2} \chi^{T}\right) \mathbf{x}_{2}\left(\underline{\mathbf{x}}_{2}\right)\right], \\
\dot{\mathbf{W}}_{o 3}=\operatorname{Proj}_{w o 3}\left[\eta_{w 3} \chi^{T} \mathbf{X}_{3}\left(\underline{\mathbf{x}}_{3}\right)\right], \\
\dot{\hat{\boldsymbol{\rho}}}_{2}=\operatorname{Proj}_{\hat{\rho}_{2}+}\left[\eta_{\rho 2}\left\|\mathbf{S}_{2}\right\|\right],
\end{gathered}
$$




$$
\begin{aligned}
& \dot{\hat{\boldsymbol{\rho}}}_{3}=\operatorname{Proj}_{\widehat{\rho}_{3}+}\left[\eta_{\rho 3} \chi^{T}\right] \text {, } \\
& \dot{\hat{\boldsymbol{\sigma}}}_{0}=\operatorname{Proj}_{\widehat{\sigma}_{0}+}\left[-\eta_{0}\left(\mathbf{S}_{2}^{T}+\gamma_{2} \boldsymbol{\chi}^{T}\right) \widehat{\mathbf{z}}\right] \text {, } \\
& \dot{\hat{\boldsymbol{\sigma}}}_{1}=\operatorname{Proj}_{\widehat{\sigma}_{1+}}\left[-\eta_{1}\left(\mathbf{S}_{2}^{T}+\gamma_{2} \boldsymbol{\chi}^{T}\right) \dot{\hat{\mathbf{z}}}\right] \text {, } \\
& \dot{\hat{\boldsymbol{\sigma}}}_{2}=\operatorname{Proj}_{\widehat{\sigma}_{2}+}\left[-\eta_{2}\left(\mathbf{S}_{2}^{T}+\gamma_{2} \chi^{T}\right) \mathbf{v}\right] \text {, } \\
& \dot{\hat{\boldsymbol{\theta}}}=\operatorname{Proj}_{\widehat{\theta}+}\left[-\eta_{\theta}\left(\mathbf{S}_{2}^{T}+\boldsymbol{\gamma}_{2} \boldsymbol{\chi}^{T}\right) \widehat{\boldsymbol{\sigma}}_{1} \widehat{\mathbf{z} h}\right], \\
& \dot{\widehat{\mathbf{m}}}_{r}=\operatorname{Proj}_{\widehat{m}_{r}+}\left[-\eta_{m r}\left(\mathbf{S}_{2}^{T}+\gamma_{2} \boldsymbol{\chi}^{T}\right) \widehat{\mathbf{m}}_{r}^{-1} \widehat{\mathbf{B}}_{m r} \boldsymbol{\delta}\right], \\
& \dot{\widehat{\mathbf{m}}}_{l}=\operatorname{Proj}_{\widehat{m}_{l}+}\left[-\eta_{m l}\left(\mathbf{S}_{2}^{T}+\boldsymbol{\gamma}_{2} \chi^{T}\right) \widehat{\mathbf{m}}_{l}^{-1} \widehat{\mathbf{B}}_{m l}(\mathbf{I}-\boldsymbol{\delta})\right], \\
& \dot{\widehat{\mathbf{B}}}_{m r}=\operatorname{Proj}_{\widehat{B}_{m r}}\left[\eta_{b r}\left(\mathbf{S}_{2}^{T}+\gamma_{2} \chi^{T}\right) \boldsymbol{\delta}\right], \\
& \dot{\widehat{\mathbf{B}}}_{m l}=\operatorname{Proj}_{\widehat{B}_{m l}-}\left[\eta_{b l}\left(\mathbf{S}_{2}^{T}+\boldsymbol{\gamma}_{2} \boldsymbol{\chi}^{T}\right)(\mathbf{I}-\boldsymbol{\delta})\right],
\end{aligned}
$$

where the projection mapping is defined as [35]

$$
\begin{gathered}
\operatorname{Proj}_{\widehat{\Omega}_{k^{+}}}(\Xi)= \begin{cases}0 & \text { if } \widehat{\Omega}_{k}=\Omega_{k \max } \text { and } \Xi>0 \\
\Xi & \text { otherwise, }\end{cases} \\
\operatorname{Proj}_{\widehat{\Omega}_{k^{ \pm}}}(\Xi)= \begin{cases}0 & \text { if } \widehat{\Omega}_{k}=\Omega_{k \max } \text { and } \Xi>0 \\
\Xi & \text { or } \widehat{\Omega}_{k}=\Omega_{k \min } \text { otherwise, } \Xi<0\end{cases} \\
\operatorname{Proj}_{\widehat{\Omega}_{-}}(\Xi)= \begin{cases}0 & \text { if } \widehat{\Omega}=\Omega_{\text {min }} \text { and } \Xi<0 \\
\Xi & \text { otherwise. }\end{cases}
\end{gathered}
$$

Substituting (58) into (57), we can obtain the following relation:

$$
\begin{aligned}
\dot{V} \leq & -\left[\begin{array}{ll}
\mathbf{S}_{1} & \mathbf{S}_{2}
\end{array}\right] \boldsymbol{\Theta}\left[\begin{array}{ll}
\mathbf{S}_{1} & \mathbf{S}_{2}
\end{array}\right]^{T}-\mathbf{K}_{1}\|\chi\|^{2}-\mathbf{K}_{2}\|\chi\| \\
& -\sum_{k=1}^{2} \frac{\left\|\lambda_{k+1}\right\|^{2}}{\varsigma_{k+1}}+\frac{5}{4} \sum_{k=1}^{2}\left\|\boldsymbol{\lambda}_{k+1}\right\|^{2} \\
& +\frac{1}{4} \sum_{k=1}^{2}\left\|\psi_{k+1}\right\|^{2}+\mathbf{g}_{n} \boldsymbol{\kappa}_{2}^{\prime}-\mathbf{g}_{n}\left(\mathbf{S}_{2}^{T}+\gamma_{2} \chi^{T}\right)\left(\boldsymbol{\sigma}_{0}-\boldsymbol{\theta} \boldsymbol{\sigma}_{1} \mathbf{h}\right) \widetilde{\mathbf{z}} \\
& +\mathbf{g}_{n}\left(\mathbf{S}_{2}^{T}+\gamma_{2} \chi^{T}\right) \boldsymbol{\sigma}_{1} \boldsymbol{\theta} \widehat{\mathbf{z}} \mathbf{h} \widetilde{\boldsymbol{\sigma}}_{0}-\mathbf{g}_{n}\left(\mathbf{S}_{2}^{T}+\gamma_{2} \chi^{T}\right) \boldsymbol{\varepsilon}_{f} \\
& +\mathbf{g}_{n}\left(-\boldsymbol{\theta} \boldsymbol{\sigma}_{0} \mathbf{h} \widetilde{\mathbf{z}}^{T}-\boldsymbol{\theta} \mathbf{h} \widehat{\mathbf{z}} \widetilde{\boldsymbol{\sigma}}_{0}-\widehat{\boldsymbol{\sigma}}_{0} \mathbf{h} \widehat{\mathbf{z}} \widetilde{\boldsymbol{\theta}}\right) \widetilde{\mathbf{z}} \\
\leq & -\mathbf{K}_{1}\|\boldsymbol{\chi}\|^{2}+\chi \boldsymbol{\Gamma}+\Delta{ }_{f},
\end{aligned}
$$

where

$$
\begin{array}{r}
\Gamma=-\mathbf{g}_{n} \gamma_{2}\left(\boldsymbol{\sigma}_{0}-\boldsymbol{\theta} \boldsymbol{\sigma}_{1} \mathbf{h}\right) \widetilde{\mathbf{z}}+\mathbf{g}_{n} \gamma_{2} \boldsymbol{\sigma}_{1} \boldsymbol{\theta} \widehat{\mathbf{z}} \mathbf{h} \widetilde{\boldsymbol{\sigma}}_{0}-\mathbf{g}_{n} \gamma_{2} \boldsymbol{\varepsilon}_{f}, \\
\Delta_{f}=\mathbf{g}_{n} \mathbf{S}_{2}^{T}\left(\left\|\sigma_{1}-\boldsymbol{\theta} \boldsymbol{\sigma}_{1} \mathbf{h}\right\|\right) \widetilde{\mathbf{z}}+\mathbf{g}_{n} \mathbf{S}_{2}^{T} \boldsymbol{\sigma}_{1} \boldsymbol{\theta} \mathbf{h}\|\widehat{\mathbf{z}}\| \widetilde{\boldsymbol{\sigma}}_{0} \\
+\mathbf{g}_{n}\left(-\boldsymbol{\theta} \boldsymbol{\sigma}_{0} \mathbf{h} \widetilde{\mathbf{z}}^{T}-\boldsymbol{\theta} \mathbf{h}\|\widehat{\mathbf{z}}\| \widetilde{\boldsymbol{\sigma}}_{0}-\widehat{\boldsymbol{\sigma}}_{0} \mathbf{h}\|\widehat{\mathbf{z}}\| \widetilde{\boldsymbol{\theta}}\right) \widetilde{\mathbf{z}}
\end{array}
$$

$$
\begin{aligned}
& +\frac{5}{4} \sum_{k=1}^{2}\left\|\lambda_{k+1}\right\|^{2}+\frac{1}{4} \sum_{k=1}^{2}\left\|\psi_{k+1}\right\|^{2} \\
& +\mathbf{g}_{n}\left\|\mathbf{S}_{2}\right\|\left\|\boldsymbol{\varepsilon}_{f}\right\|+\mathbf{g}_{n} \boldsymbol{\kappa}_{2}^{\prime} \\
& =-\mathbf{g}_{n}\left(\left\|\mathbf{S}_{2}\right\|-\frac{\left\|\sigma_{1}-\boldsymbol{\theta} \boldsymbol{\sigma}_{1} \mathbf{h}\right\|}{2}\|\widetilde{\mathbf{z}}\|\right)^{2} \\
& -\frac{\mathbf{g}_{n}\left\|\boldsymbol{\sigma}_{1}-\boldsymbol{\theta} \boldsymbol{\sigma}_{1} \mathbf{h}\right\|}{4}\|\widetilde{\mathbf{z}}\|^{2} \\
& +\mathbf{g}_{n}\left(\left\|\mathbf{S}_{2}\right\|^{2}-\frac{\left\|\boldsymbol{\sigma}_{1} \boldsymbol{\theta} \mathbf{h} \widehat{\mathbf{z}}\right\|^{2}}{2}\left\|\widetilde{\boldsymbol{\sigma}}_{0}\right\|^{2}\right)^{2} \\
& -\mathbf{g}_{n}\left\|\mathbf{S}_{2}\right\|^{2}-\frac{\mathbf{g}_{n}\left\|\boldsymbol{\sigma}_{1} \boldsymbol{\theta} \mathbf{h} \hat{\mathbf{z}}\right\|^{2}}{4}\left\|\widetilde{\boldsymbol{\sigma}}_{0}\right\|^{2} \\
& -\mathbf{g}_{n} \boldsymbol{\theta}\left\|\boldsymbol{\sigma}_{0}\right\| \mathbf{h}\left(\|\widetilde{\mathbf{z}}\|+\frac{1}{2\left\|\boldsymbol{\sigma}_{0}\right\|}\left\|\widetilde{\boldsymbol{\sigma}}_{0}\right\|\right)^{2} \\
& +\frac{\mathbf{g}_{n} \boldsymbol{\theta}}{4\left\|\boldsymbol{\sigma}_{0}\right\|}\left\|\widetilde{\boldsymbol{\sigma}}_{0}\right\|^{2}-\mathbf{g}_{n}\left(\|\widetilde{\boldsymbol{\theta}}\|^{2}+\frac{\left\|\widehat{\boldsymbol{\sigma}}_{0} \mathbf{h} \widehat{\mathbf{z}}\right\|^{2}}{2}\|\widetilde{\mathbf{z}}\|^{2}\right) \\
& +\frac{\left\|\widehat{\boldsymbol{\sigma}}_{0} \mathbf{h} \widehat{\mathbf{z}}\right\|^{2}}{4}\|\widetilde{\mathbf{z}}\|^{2}+\frac{5}{4} \sum_{k=1}^{2}\left\|\lambda_{k+1}\right\|^{2} \\
& +\frac{1}{4} \sum_{k=1}^{2}\left\|\psi_{k+1}\right\|^{2}+\mathbf{g}_{n}\left\|\mathbf{S}_{2}\right\|\left\|\varepsilon_{f}\right\|+\mathbf{g}_{n} \boldsymbol{\kappa}_{2}^{\prime},
\end{aligned}
$$

and the positive definite matrix $\boldsymbol{\Theta}_{i}$ is described as

$$
\boldsymbol{\Theta}=\left[\begin{array}{cc}
\left(\mathbf{c}_{1}-1\right) & \mathbf{0} \\
\gamma_{1} & \left(\mathbf{c}_{2}-1\right)+\gamma_{2}
\end{array}\right] .
$$

Then (60) can be written as

$$
\begin{aligned}
\dot{V}= & -\frac{1}{2} \chi^{T}\left(2 \mathbf{K}_{1}-\frac{\mathbf{I}}{\mu^{2}}\right) \chi-\frac{1}{2}\left(\frac{\mathbf{I}}{\mu} \chi-\mu \Gamma\right)^{T}\left(\frac{\mathbf{I}}{\mu} \chi-\mu \Gamma\right) \\
& +\frac{1}{2} \mu^{2}\|\mathbf{\Gamma}\|^{2}+\Delta_{f} \\
\leq & -\frac{1}{2} \chi^{T} \mathbf{Q} \chi+\frac{1}{2} \mu^{2}\|\mathbf{\Gamma}\|^{2}+\Delta_{f}
\end{aligned}
$$

where $\mathbf{Q}=\left(2 \mathbf{K}_{1}-\mathbf{I} / \mu^{2}\right), \mathbf{K}_{1}>\mathbf{I} / 2 \mu^{2}$, and $\mu$ is a positive constant. By integrating both sides of (64) from $t=0$ to $t=$ $\infty$, we obtain the following inequality:

$$
\begin{aligned}
V(T) \leq & V(0)-\frac{1}{2} \lambda_{\min }(\mathbf{Q}) \int_{0}^{T}\|\boldsymbol{\chi}\|^{2} d t \\
& +\frac{1}{2} \mu^{2} \int_{0}^{T}\|\boldsymbol{\Gamma}\|^{2} d t \\
& +\mathbf{g}_{n} \int_{0}^{T}\left(\left\|\mathbf{S}_{2}\right\|-\frac{\left\|\boldsymbol{\sigma}_{1} \boldsymbol{\theta} \mathbf{h} \widehat{\mathbf{z}}\right\|^{2}}{2}\left\|\widetilde{\boldsymbol{\sigma}}_{0}\right\|\right)^{2} d t
\end{aligned}
$$




$$
\begin{aligned}
& +\frac{1}{4} \int_{o}^{T} \frac{\mathbf{g}_{n} \boldsymbol{\theta}}{\left\|\boldsymbol{\sigma}_{0}\right\|}\left\|\tilde{\boldsymbol{\sigma}}_{0}\right\|^{2} d t+\frac{1}{4} \int_{0}^{T}\left\|\widehat{\boldsymbol{\sigma}}_{0} \mathbf{h} \widehat{\mathbf{z}}\right\|^{2}\|\widetilde{\mathbf{z}}\|^{2} d t \\
& +\int_{0}^{T}\left(\frac{5}{4} \sum_{k=1}^{2}\left\|\boldsymbol{\lambda}_{k+1}\right\|^{2}+\frac{1}{4} \sum_{k=1}^{2}\left\|\psi_{k+1}\right\|^{2}\right) d t+\Delta,
\end{aligned}
$$

for all $0 \leq T<\infty$ with $\Delta=\mathbf{g}_{n}\left\|\mathbf{S}_{2}\right\|\left\|\boldsymbol{\varepsilon}_{f}\right\|+$ $\mathbf{g}_{n} \boldsymbol{\kappa}_{2}^{\prime}$. This implies all the states and signals are bounded. Finally, we can conclude that $\Gamma \in L_{2}[0, \infty) \cap L_{\infty}[0, \infty)$, $\left\|\mathbf{S}_{2}\right\|-\left(\left\|\boldsymbol{\sigma}_{1} \boldsymbol{\theta} \mathbf{h} \widehat{\mathbf{z}}\right\|^{2} / 2\right)\left\|\tilde{\boldsymbol{\sigma}}_{0}\right\| \quad \in \quad L_{2}[0, \infty) \cap L_{\infty}[0, \infty)$, $\left(\mathbf{g}_{n} \boldsymbol{\theta} /\left\|\boldsymbol{\sigma}_{0}\right\|\right)\left\|\tilde{\boldsymbol{\sigma}}_{0}\right\|^{2} \in L_{2}[0, \infty) \cap L_{\infty}[0, \infty),\left\|\widehat{\boldsymbol{\sigma}}_{0} \mathbf{h} \widehat{\mathbf{z}}\right\|^{2}\|\widetilde{\mathbf{z}}\|^{2} \in$ $L_{2}[0, \infty) \cap L_{\infty}[0, \infty), \sum_{k=1}^{2}\left\|\lambda_{k+1}\right\|^{2} \in L_{2}[0, \infty) \cap$ $L_{\infty}[0, \infty), \sum_{k=1}^{2}\left\|\psi_{k+1}\right\|^{2} \in L_{2}[0, \infty) \cap L_{\infty}[0, \infty)$, and $\Delta \in$ $L_{2}[0, \infty) \cap L_{\infty}[0, \infty)$. Then, $\mathbf{S}_{i} \rightarrow \mathbf{0}, \boldsymbol{\chi} \rightarrow \mathbf{0 ,} \widetilde{\mathbf{W}}_{o i} \rightarrow$ $\mathbf{0}, \widetilde{\boldsymbol{\rho}}_{i} \rightarrow \mathbf{0}, \widetilde{\boldsymbol{\sigma}}_{0} \rightarrow \mathbf{0}, \widetilde{\boldsymbol{\sigma}}_{1} \rightarrow \mathbf{0}, \widetilde{\boldsymbol{\sigma}}_{2} \rightarrow \mathbf{0}, \widetilde{\boldsymbol{\theta}} \rightarrow \mathbf{0}, \widetilde{\mathbf{m}}_{r} \rightarrow \mathbf{0}$, $\widetilde{\mathbf{m}}_{l} \rightarrow \mathbf{0}, \widetilde{\mathbf{B}}_{m r} \rightarrow \mathbf{0}$, and $\widetilde{\mathbf{B}}_{m l} \rightarrow \mathbf{0}$ as $t \rightarrow \infty$ by Barbalat's Lemma [30].

\section{Experimental Example}

The experiments to evaluate the proposed control scheme using the Scorbot robot system are described in this section. A photograph of the Scorbot robot is given in Figure 2, where the deadzone occurs in the timing belt. We select only two links (upper arm = link1 and forearm = link2) among the four links of the Scorbot robot manipulator to simplify the verification process of our position control. From (1) to (3), the dynamic equations for the two DOF (degree of freedom) links of the Scorbot robot manipulator are described as

$$
\begin{gathered}
\mathbf{M}(\mathbf{q}) \ddot{\mathbf{q}}+\mathbf{C}(\mathbf{q}, \dot{\mathbf{q}}) \dot{\mathbf{q}}+\mathbf{G}(\mathbf{q})+\mathbf{T}_{f}(\mathbf{q}, \dot{\mathbf{q}})+\mathbf{T}_{L}=\boldsymbol{\tau}, \\
\boldsymbol{\tau}=\mathbf{n k}_{t} \mathbf{i}, \\
\mathbf{L}_{m} \frac{d \mathbf{i}}{d t}+\mathbf{R}_{m} \mathbf{i}+\mathbf{k}_{b} \dot{\mathbf{q}}=\mathbf{V},
\end{gathered}
$$

where

$$
\begin{gathered}
\mathbf{M}(\mathbf{q})=\left[\begin{array}{ll}
M_{11} & M_{12} \\
M_{21} & M_{22}
\end{array}\right], \\
M_{11}=\left(m_{1}+m_{2}\right) L_{1}^{2}+m_{2} L_{2}^{2}+2 m_{2} L_{1} L_{2} \cos \left(q_{2}\right), \\
M_{12}=m_{2} L_{2}^{2}+L_{1} L_{2} m_{2} \cos \left(q_{2}\right), \\
M_{21}=m_{2} L_{2}^{2}+L_{1} L_{2} m_{2} \cos \left(q_{2}\right), \\
M_{22}=m_{2} L_{2}^{2}, \\
\mathbf{C}(\mathbf{q}, \dot{\mathbf{q}})=\left[\begin{array}{c}
\left.-m_{2} L_{1} L_{2} \sin \left(q_{2}\right) \dot{q}_{2}^{2}-2 m_{2} L_{1} L_{2} \sin \left(q_{2}\right) \dot{q}_{1} \dot{q}_{2}\right] \\
m_{2} L_{1} L_{2} \sin \left(q_{2}\right) \dot{q}_{1}^{2}
\end{array}\right], \\
\mathbf{G}(\mathbf{q})=\left[\begin{array}{c}
m_{2} L_{2} g \cos \left(q_{1}+q_{2}\right)+\left(m_{1}+m_{2}\right) L_{1} g \cos \left(q_{1}\right) \\
m_{2} L_{2} g \cos \left(q_{1}+q_{2}\right)
\end{array}\right], \\
\mathbf{F}_{f}(\mathbf{q}, \dot{\mathbf{q}})=\left[\begin{array}{l}
\sigma_{01} z_{1}+\sigma_{11} \dot{z}_{1}+\sigma_{21} \dot{q}_{1} \\
\sigma_{02} z_{2}+\sigma_{12} \dot{z}_{2}+\sigma_{22} \dot{q}_{2}
\end{array}\right] .
\end{gathered}
$$

The parameter values chosen for each link and actuator are represented in Table 1 . The sine wave joint and circle motions of the end effector are chosen to be the desired trajectory commands. The sine wave is chosen to be $\mathbf{q}_{d}(t)=$ $0.005 \sin (1.2566 t)$ (rad). The direct kinematics for a circle trajectory in a task space is given by

$$
\boldsymbol{\Phi}(\mathbf{q})=\left[\begin{array}{l}
L_{1} \sin \left(q_{1}\right)+L_{2} \sin \left(q_{1}+q_{2}\right) \\
L_{1} \cos \left(q_{1}\right)+L_{2} \cos \left(q_{1}+q_{2}\right)
\end{array}\right] .
$$

Then, the desired end-effector trajectory of the manipulator becomes

$$
\mathbf{Y}_{d}(t)=\left[\begin{array}{l}
x_{d} \\
y_{d}
\end{array}\right]=\left[\begin{array}{l}
x_{c}+R \cos (\omega \times t) \\
y_{c}+R \sin (\omega \times t)
\end{array}\right]
$$

where $x_{c}=y_{c}=-0.1 \mathrm{~m}, R=2.5 \mathrm{~mm}$, and $\omega=0.45 \mathrm{rad} / \mathrm{sec}$. This trajectory makes the manipulator tip trace a circle in the $x_{0}-y_{0}$ plane with a radius of $R=2.5 \mathrm{~mm}$. The desired trajectory $\mathbf{Y}_{d}$ was translated to the corresponding joint space desired position trajectory $\mathbf{q}_{d}$ via the inverse kinematics of the simulated two DOF links manipulators:

$$
\begin{aligned}
& \mathbf{q}_{d}=\boldsymbol{\Phi}^{-1}\left(\mathbf{Y}_{d}\right)=\left[\begin{array}{l}
q_{d 1} \\
q_{d 2}
\end{array}\right] \\
& =\left[\begin{array}{c}
\tan ^{-1}\left(\frac{y_{d} / x_{d}-\tan ^{-1}\left(L_{2} \sin \left(q_{d 2}\right)\right)}{L_{1}+L_{2} \cos \left(q_{d 2}\right)}\right) \\
\tan ^{-1}\left(\frac{1-\left(x_{d}^{2}+y_{d}^{2}-L_{1}^{2}-L_{2}^{2}\right)^{2} /\left(2 L_{1} L_{2}\right)^{2}}{\left(x_{d}^{2}+y_{d}^{2}-L_{1}^{2}-L_{2}^{2}\right) /\left(2 L_{1} L_{2}\right)}\right)
\end{array}\right] .
\end{aligned}
$$

The design parameters of the controller are given in Table 2. The fuzzy membership functions for the linkl are chosen to be

$$
\begin{aligned}
\mu_{F_{1}^{1}} & =\frac{1}{1+\exp \left[\left(S_{11}-4.5 \times 10^{-3}\right)^{2}\right]}, \\
\mu_{F_{1}^{2}} & =\exp \left[-\left(S_{11}-3 \times 10^{-3}\right)^{2}\right], \\
\mu_{F_{1}^{3}} & =\exp \left[-\left(S_{11}-1.5 \times 10^{-3}\right)^{2}\right], \\
\mu_{F_{1}^{4}} & =\exp \left[-\left(S_{11}+0 \times 10^{-3}\right)^{2}\right], \\
\mu_{F_{1}^{5}} & =\exp \left[-\left(S_{11}+1.5 \times 10^{-3}\right)^{2}\right], \\
\mu_{F_{1}^{6}} & =\exp \left[-\left(S_{11}+3 \times 10^{-3}\right)^{2}\right], \\
\mu_{F_{1}^{7}} & =\frac{1}{1+\exp \left[\left(S_{11}+4.5 \times 10^{-3}\right)^{2}\right]} .
\end{aligned}
$$

The second inputs of the fuzzy system in linkl are chosen to be $\dot{S}_{11}$ and the membership functions are the same as the above 


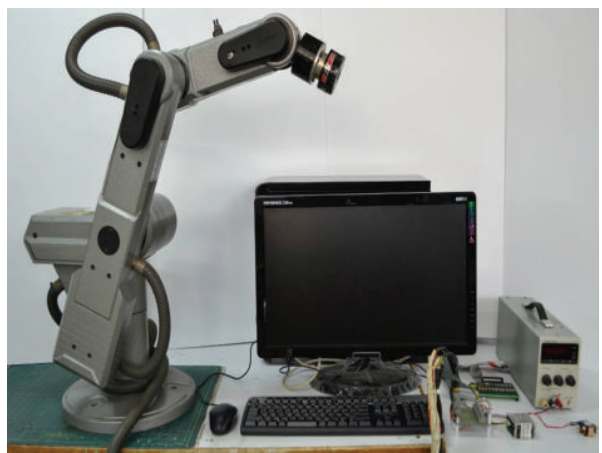

(a)

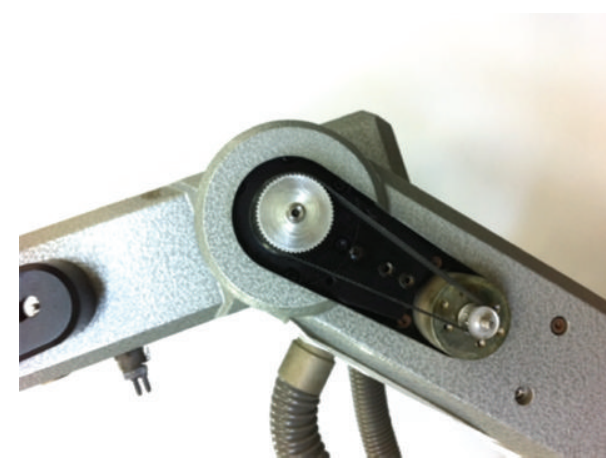

(b)

Figure 2: Photograph of the Scorbot robot system: (a) manipulator; (b) timing belt in the actuator.
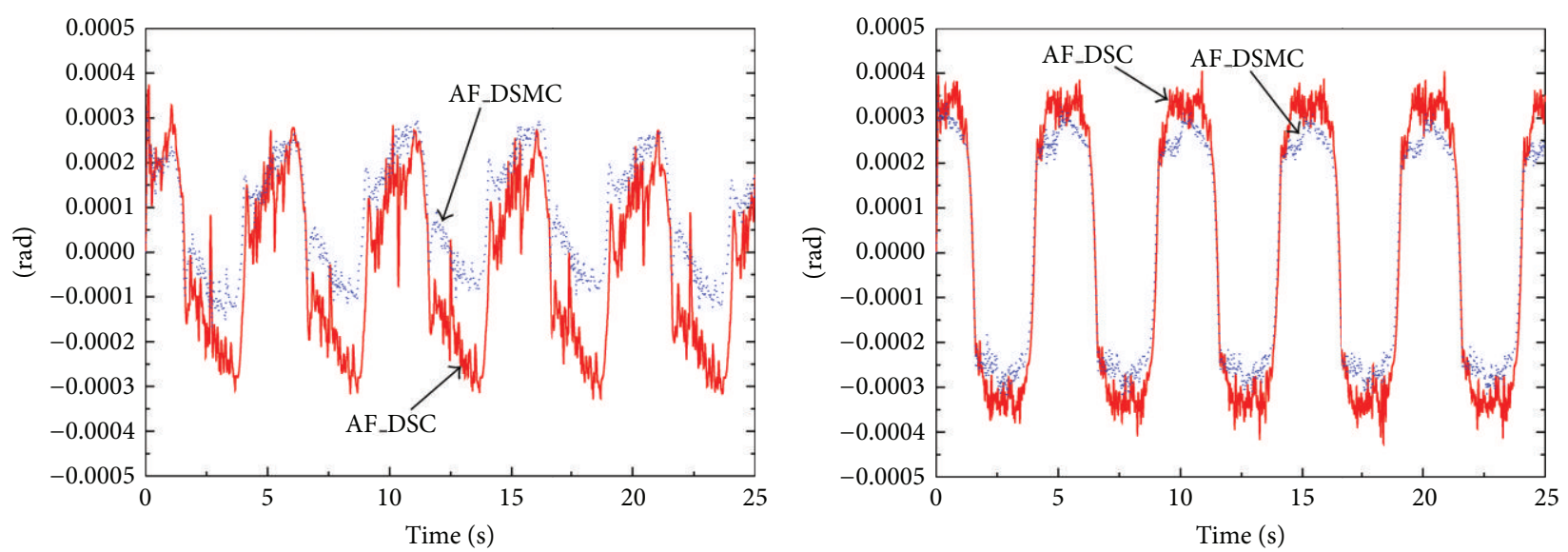

(a)

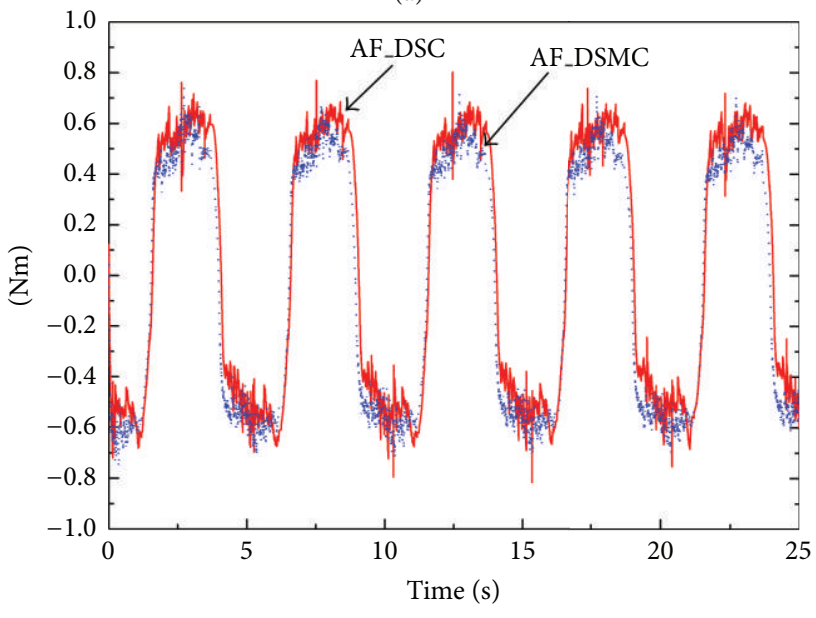

(c) (b)

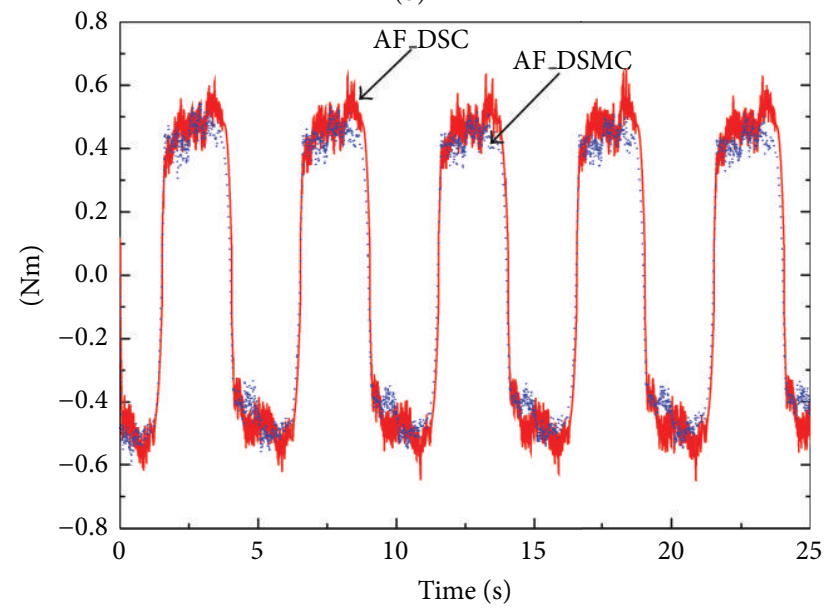

(d)

FIGURE 3: Experimental results of AF_DSC and AF_DSMC systems for the sine-wave command: (a) tracking errors in link1; (b) tracking errors in link2; (c) $u_{d 1}$; (d) $u_{d 2}$.

expressions. The fuzzy membership functions for link2 are chosen to be

$$
\begin{aligned}
\mu_{F_{2}^{1}} & =\frac{1}{1+\exp \left[\left(S_{21}-9 \times 10^{-4}\right)^{2}\right]} \\
\mu_{F_{2}^{2}} & =\exp \left[-\left(S_{21}-6 \times 10^{-4}\right)^{2}\right] \\
\mu_{F_{2}^{3}} & =\exp \left[-\left(S_{21}-3 \times 10^{-4}\right)^{2}\right]
\end{aligned}
$$

$$
\begin{aligned}
\mu_{F_{2}^{4}} & =\exp \left[-\left(S_{21}+0 \times 10^{-4}\right)^{2}\right], \\
\mu_{F_{2}^{5}} & =\exp \left[-\left(S_{21}+3 \times 10^{-4}\right)^{2}\right], \\
\mu_{F_{2}^{6}} & =\exp \left[-\left(S_{21}+6 \times 10^{-4}\right)^{2}\right], \\
\mu_{F_{2}^{7}} & =\frac{1}{1+\exp \left[\left(S_{21}+9 \times 10^{-4}\right)^{2}\right]} .
\end{aligned}
$$




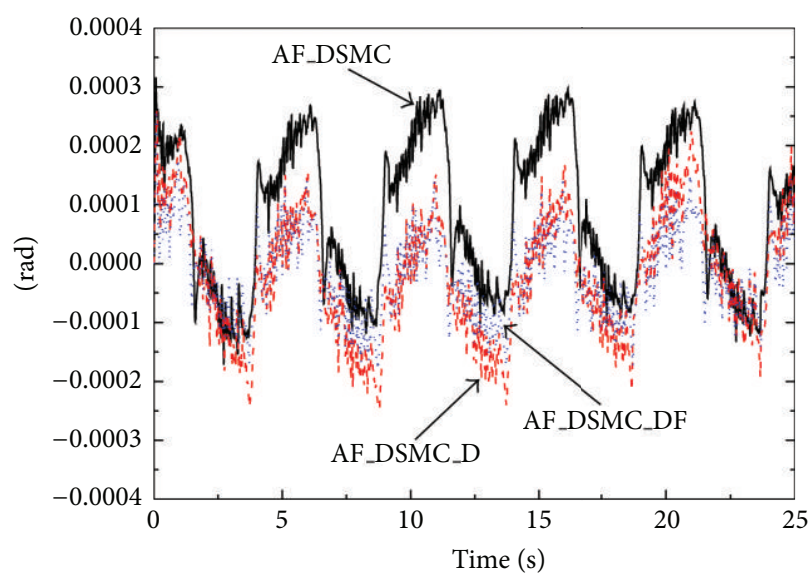

(a)

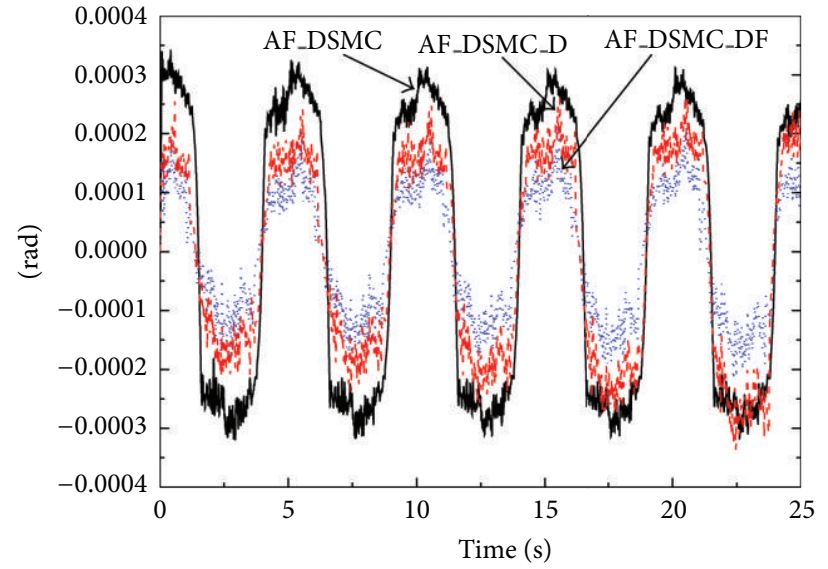

(b)

Figure 4: Experimental results of AF_DSMC, AF_DSMC_D, and AF_DSMC_DF systems for the sine command: (a) tracking errors in link1; (b) tracking errors in link2.

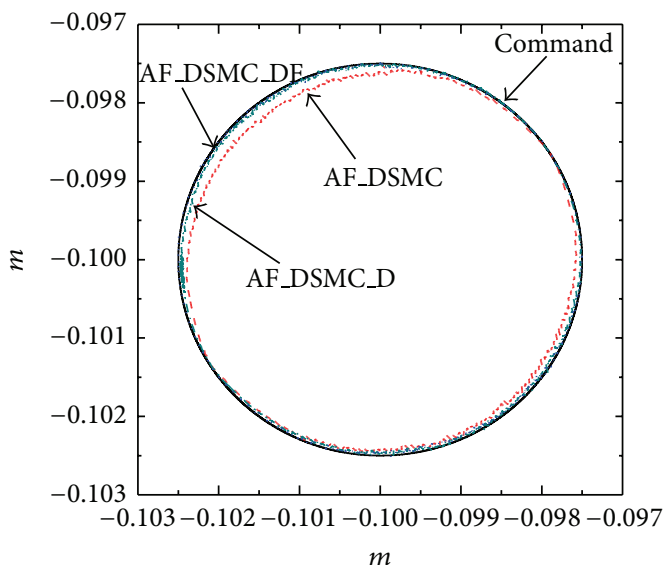

(a)

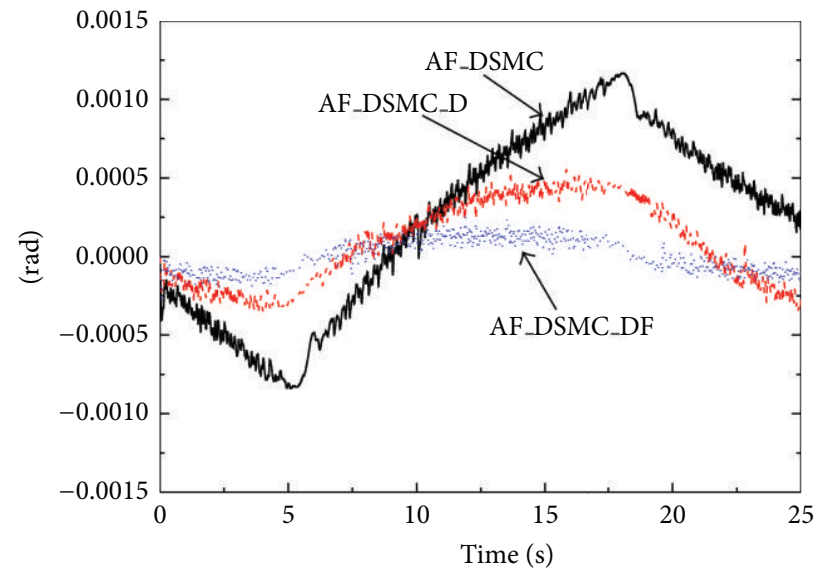

(b)

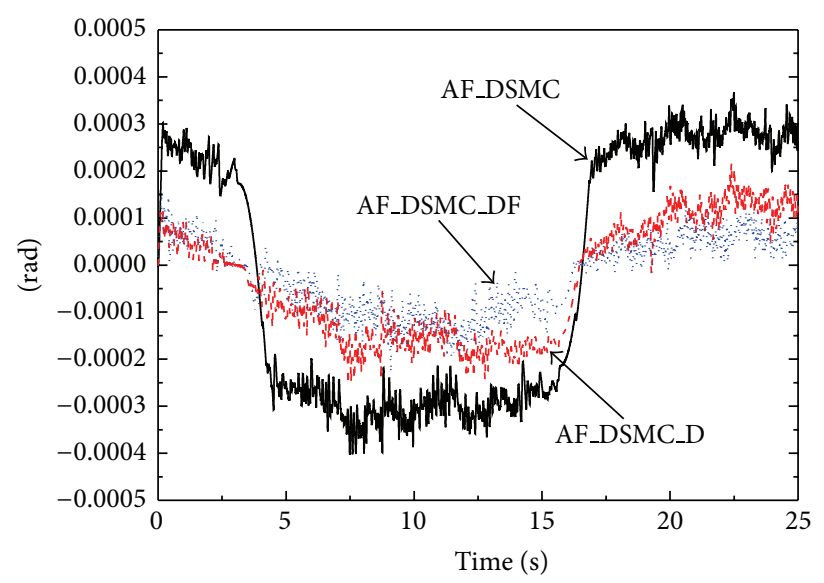

(c)

Figure 5: Experimental results of AF_DSMC, AF_DSMC_D, and AF_DSMC_DF systems for the circle command: (a) tracking results; (b) tracking errors in link1; (c) tracking errors in link2. 
TABLE 1: Manipulator parameters.

\begin{tabular}{llc}
\hline Symbol & Parameter & Quantity \\
\hline$m_{1}, m_{2}$ & Mass of links 1 and 2 & $12.1 \mathrm{~kg}, 3.59 \mathrm{~kg}$ \\
$L_{1}, L_{2}$ & Mass of links 1 and 2 & $0.3 \mathrm{~m}, 0.41 \mathrm{~m}$ \\
$F_{s 1}, F_{s 2}$ & Stiction level of joints 1 and 2 & $0.063 \mathrm{Nm}, 0.0648 \mathrm{Nm}$ \\
$F_{c 1}, F_{c 2}$ & Coulomb friction of joints 1 and 2 & $0.061 \mathrm{Nmsec} / \mathrm{rad}, 0.06 \mathrm{Nmsec} / \mathrm{rad}$ \\
$v_{s 1}, v_{s 2}$ & Stibeck velocity of joints 1 and 2 & $0.00075 \mathrm{rad} / \mathrm{sec}, 0.00063 \mathrm{rad} / \mathrm{sec}$ \\
$\sigma_{01}, \sigma_{02}$ & Bristle stiffness of joints 1 and 2 & $5400 \mathrm{Nm} / \mathrm{rad}, 8700 \mathrm{Nm} / \mathrm{rad}$ \\
$\sigma_{11}, \sigma_{12}$ & Presliding damping of joints 1 and 2 & $5.4 \mathrm{Nmsec} / \mathrm{rad}, 6.2 \mathrm{Nmsec} / \mathrm{rad}$ \\
$\sigma_{21}, \sigma_{22}$ & Sliding damping of joints 1 and 2 & $10.2 \mathrm{Nmsec} / \mathrm{rad}, 10.8 \mathrm{Nmsec} / \mathrm{rad}$ \\
$\theta_{1}, \theta_{2}$ & Transient friction parameter of joints $1 \mathrm{and} 2$ & $0.87,0.9$ \\
$m_{r 1}, m_{l 1}$ & Slope of deadzone of joint 1 & 1,1 \\
$m_{r 2}, m_{l 2}$ & Slope of deadzone of joint 2 & 1,1 \\
$B_{r 1}, B_{l 1}$ & Deadzone width of joint 1 & $0.28,-0.28$ \\
$B_{r 2}, B_{l 2}$ & Deadzone width of joint 1 & $0.25,-0.25$ \\
$n_{i}$ & Gear ratio of reduction gear & 65.5 \\
$L_{m i}$ & Inductance of motor & $0.6292 \mathrm{mH}$ \\
$R_{m i}$ & Resistance of motor & $0.8294 \Omega$ \\
$k_{t i}$ & Torque constant & $0.0182 \mathrm{Nm} / \mathrm{A}$ \\
$k_{b i}$ & Back emf constant & $0.0182 \mathrm{~V} / \mathrm{rad} / \mathrm{sec}$ \\
\hline
\end{tabular}

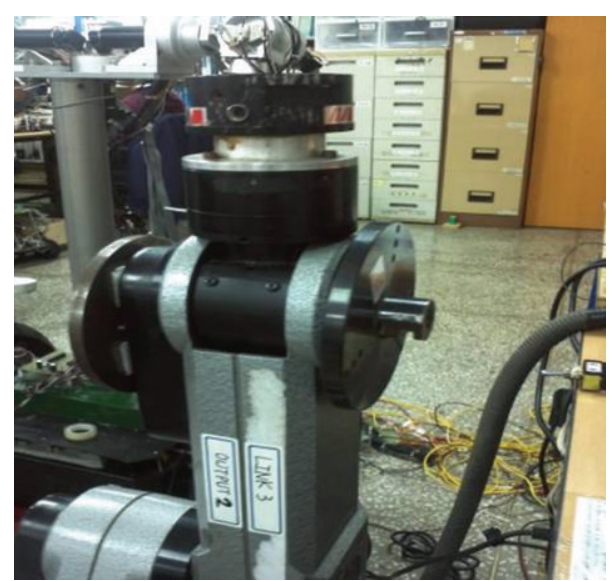

FIGURE 6: Photograph of the link2 with attached mass.

The second inputs of the fuzzy system in link2 are chosen to be $\dot{S}_{21}$ and the membership functions are the same as the above expressions. The designed controllers generated in the computer are implemented in the MATLAB RTI system using an MF624 board (Humusoft) [38]. The control signals were transferred into the DC servomotor of the Scorbot robot through the servodrive. The sample frequency was set to1 $\mathrm{kHz}$.

In (18), the term $\mathbf{f}_{3}\left(\underline{x}_{3}\right)$ is taken to be uncertain and is not approximated by the fuzzy system since this uncertainty can be compensated for by adding a SMC in (56). Firstly, we designed two controllers to evaluate the performance of the proposed system: the proposed adaptive fuzzy DSC system with SMC (AF_DSMC) and the conventional fuzzy DSC system without SMC (AF_DSC). The experimental results are shown in Figure 3. The tracking
TABLE 2: Controller parameters.

\begin{tabular}{lc}
\hline Parameter & Quantity \\
\hline$c_{11}, c_{12}$ & 120,20 \\
$c_{21}, c_{22}$ & 80,10 \\
$\gamma_{11}, \gamma_{12}$ & $0.2,0.2$ \\
$\gamma_{21}, \gamma_{22}$ & $0.2,0.2$ \\
$K_{11}, K_{12}$ & $0.12,0.12$ \\
$K_{21}, K_{22}$ & $0.12,0.12$ \\
$\widehat{\rho}_{12}, \widehat{\rho}_{22}$ & $0.2,0.2$ \\
$\tau_{12}, \tau_{13}$ & $0.5,0.5$ \\
$\tau_{22}, \tau_{23}$ & $0.05,0.05$ \\
\hline
\end{tabular}

TABLE 3: RMS tracking error for the sine command.

\begin{tabular}{lcccc}
\hline \multirow{2}{*}{ Control system } & \multicolumn{2}{c}{ RMS error (rad) } & \multicolumn{2}{c}{ RMS error (\%) } \\
& Link1 & Link2 & Link1 & Link2 \\
\hline AF_DSC & $1.83 \times 10^{-4}$ & $2.95 \times 10^{-4}$ & 100 & 100 \\
AF_DSC & $1.51 \times 10^{-4}$ & $2.43 \times 10^{-4}$ & 82.5 & 82.4 \\
AF_DSMC_D & $1.05 \times 10^{-4}$ & $1.69 \times 10^{-4}$ & 57.4 & 57.3 \\
AF_DSMC_DF & $0.75 \times 10^{-4}$ & $1.06 \times 10^{-4}$ & 41 & 36 \\
\hline
\end{tabular}

errors of AF_DSMC system in Figures 3(a) and 3(b) are significantly lower than those of the AF_DSC due to the addition of a SMC into the controller in spite of the similar control inputs as shown in Figures 3(c) and 3(d). These results validate the general robustness property of a SMC. These experimental results show that the proposed dynamic surface control combined with a SMC has a superior performance than that of the conventional DSC system.

Next, we designed two additional control systems: an adaptive fuzzy dynamic surface sliding mode controller 


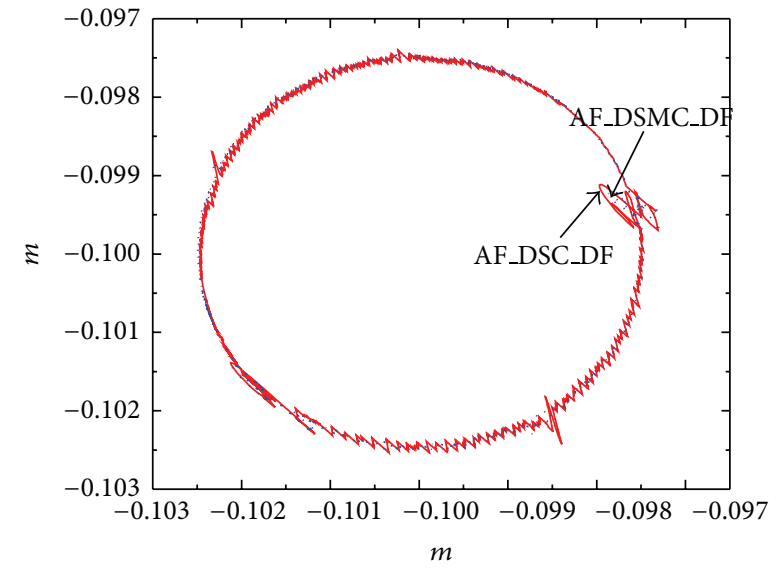

(a)

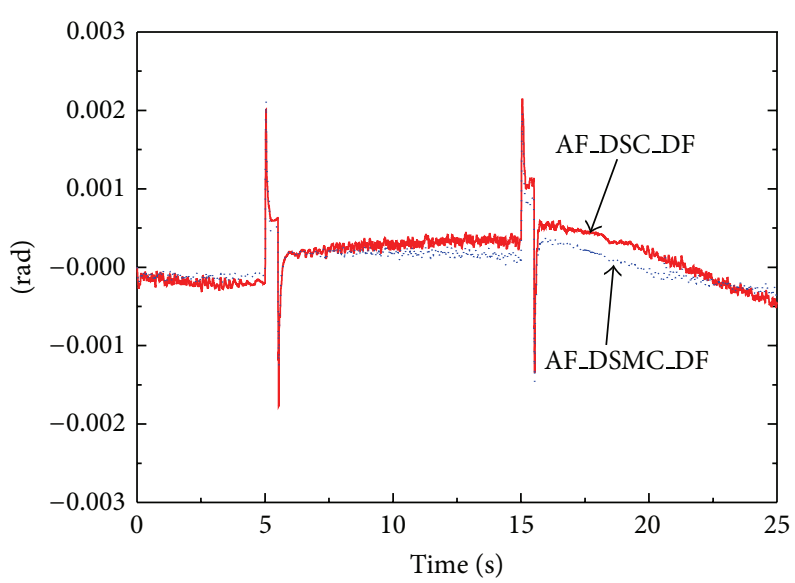

(b)

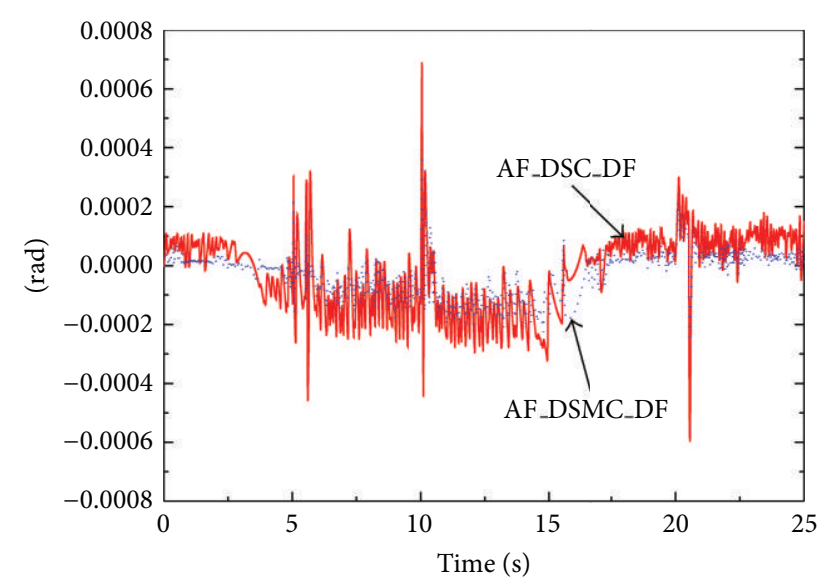

(c)

Figure 7: Experimental results of AF_DSC_DF and AF_DSMC_DF systems for the circle command against disturbances: (a) tracking results; (b) tracking errors in link1; (c) tracking errors in link2.

with deadzone compensation alone (AF_DSMC_D) and an adaptive fuzzy dynamic surface sliding mode controller with both deadzone and friction compensation (AF_DSMC_DF). Experimental results for sine-wave command inputs are shown in Figure 4, where it can be seen that the proposed AF_DSMC_DF has much better tracking performance than those of deadzone compensation alone (AD_DSMC_D) and no compensation (AF_DSMC). In Figure 5, the estimated results for the fuzzy output weights, uncertainty, friction, and deadzone parameters are given for a sine-wave command input.

To quantitatively evaluate the tracking performance of each control system, the RMS tracking errors for a sine-wave command are summarized in Table 3. The size of the RMS tracking error is decreased by as much as $36 \%$ in the proposed control scheme compared to that of the AF_ DSC system.

The circle trajectory tracking results are represented in Figure 6, where it can be seen that, due to compensation of friction and deadzone, the circle tracking errors in the AF_DSMC_DF system are much lower than the errors of the AF_DSMC and AF_DSMC_D systems. To evaluate robustness to external disturbance, an additional mass of $1.8 \mathrm{~kg}$ was attached to link2 as shown in Figure 7 and pulse disturbances of 2 and $0.2 \mathrm{~V}$ for $0.5 \mathrm{sec}$ of pulse widths were applied to each motor drive at $5,10,15$, and $20 \mathrm{sec}$, respectively. The two control schemes with the deadzone and friction compensators with SMC (AFE_DSMC_DF) and without SMC (AFE_DSC_DF) were evaluated. As can be seen in Figure 7, the proposed AFE _DSMC_DF system is more robust to disturbance because of the SMC.

The RMS tracking errors for a circle command are summarized in Table 4, where the magnitude of the RMS tracking error is decreased as much as $16.5 \%$ in the proposed control scheme compared to that of the AF_ DMSC system.

\section{Conclusion}

In this paper, a nonmodel based dynamic surface sliding mode control scheme has been developed to provide significantly enhanced position tracking performance of a MIMO robot manipulator system in the presence of both deadzone and friction on the part of the actuator. An adaptive fuzzy system is considered to approximate the unknown uncertainty of the complex manipulator dynamics. To enforce the 
TABLE 4: RMS tracking error for the circle command.

\begin{tabular}{lcccc}
\hline \multirow{2}{*}{ Control system } & \multicolumn{2}{c}{ RMS error (rad) } & \multicolumn{2}{c}{ RMS error (\%) } \\
& Link1 & Link2 & Link1 & Link2 \\
\hline AF_DSMC & $6.30 \times 10^{-4}$ & $2.66 \times 10^{-4}$ & 100 & 55.3 \\
AF_DSMC_D & $2.84 \times 10^{-4}$ & $1.26 \times 10^{-4}$ & 45.1 & 47.4 \\
AF_DSMC_DF & $1.04 \times 10^{-4}$ & $0.80 \times 10^{-4}$ & 16.5 & 30 \\
AF_DSC_DF & $3.66 \times 10^{-4}$ & $1.31 \times 10^{-4}$ & 58.1 & 49.3 \\
$\begin{array}{l}\text { (disturbance) } \\
\begin{array}{l}\text { AF_DSMC_DF } \\
\text { (disturbance) }\end{array}\end{array}$ & $2.69 \times 10^{-4}$ & $0.81 \times 10^{-4}$ & 42.7 & 30.5 \\
\hline
\end{tabular}

robustness of the DSC scheme, a SMC is also considered as well to introduce estimators for the unknown parameters of the Elastoplastic friction model and deadzone. The recursive steps of the DSC design procedure provide adaptive laws for the controller, friction, and deadzone estimators. As an example application, a Scorbot robot manipulator in the presence of joint friction and deadzone was tested. The favorable positioning performance of the proposed control scheme has been experimentally validated to effectively compensate for deadzone, friction, and uncertainty.

\section{Acknowledgment}

This research was supported by The Ministry of Trade, Industry and Energy (MOTIE), Republic of Korea, under the Human Resources Development Program for Specialized Environment Navigation/Localization Technology Research Center support program supervised by the National IT Industry Promotion Agency (NIPA) (H1502-13-1001).

\section{References}

[1] M. Kristic, I. Kanellakopoulos, and P. V. Kokotovic, Nonlinear and Adaptive Control Design, John Wiley \& Sons, New York, NY, USA, 1995.

[2] Y. Zhang, B. Fidan, and P. A. Ioannou, "Backstepping control of linear time-varying systems with known and unknown parameters," IEEE Transactions on Automatic Control, vol. 48, no. 11, pp. 1908-1925, 2003.

[3] S. Jagannathan and F. L. Lewis, "Robust backstepping control of a class of nonlinear systems using fuzzy logic," Information Sciences, vol. 123, no. 3-4, pp. 223-240, 2000.

[4] Y. Yang, G. Feng, and J. Ren, "A combined backstepping and small-gain approach to robust adaptive fuzzy control for strictfeedback nonlinear systems," IEEE Transactions on Systems, Man, and Cybernetics A, vol. 34, no. 3, pp. 406-420, 2004.

[5] Y. Li, S. Qiang, X. Zhuang, and O. Kaynak, "Robust and adaptive backstepping control for nonlinear systems using RBF neural networks," IEEE Transactions on Neural Networks, vol. 15, no. 3 , pp. 693-701, 2004.

[6] C. Kwan and F. L. Lewis, "Robust backstepping control of nonlinear systems using neural networks," IEEE Transactions on Systems, Man, and Cybernetics A, vol. 30, no. 6, pp. 753-766, 2000.

[7] D. Swaroop, J. K. Hedrick, P. P. Yip, and J. C. Gerdes, "Dynamic surface control for a class of nonlinear systems," IEEE Transactions on Automatic Control, vol. 45, no. 10, pp. 1893-1899, 2000.
[8] J. K. Hedrick and P. P. Yip, "Multiple sliding surface control: theory and application," Journal of Dynamic Systems, Measurement and Control, vol. 122, no. 4, pp. 586-593, 2000.

[9] S. Tong, Y. Li, G. Feng, and T. Li, "Observer-based adaptive fuzzy backstepping dynamic surface control for a class of nonlinear systems with unknown time delays," IET Control Theory \& Applications, vol. 5, no. 12, pp. 1426-1438, 2011.

[10] D. Wang and J. Huang, "Neural network-based adaptive dynamic surface control for a class of uncertain nonlinear systems in strict-feedback form," IEEE Transactions on Neural Networks, vol. 16, no. 1, pp. 195-202, 2005.

[11] S. J. Yoo, J. B. Park, and Y. H. Choi, "Adaptive dynamic surface control of flexible-joint robots using self-recurrent wavelet neural networks," IEEE Transactions on Systems, Man, and Cybernetics B, vol. 36, no. 6, pp. 1342-1355, 2006.

[12] T. P. Zhang and S. S. Ge, "Adaptive dynamic surface control of nonlinear systems with unknown dead zone in pure feedback form," Automatica, vol. 44, no. 7, pp. 1895-1903, 2008.

[13] G. Tao and P. V. Kokotović, "Adaptive control of plants with unknown dead-zones," IEEE Transactions on Automatic Control, vol. 39, no. 1, pp. 59-68, 1994.

[14] C. C. de Wit, H. Olsson, K. J. Astrom, and P. Lischinsky, "A new model for control of systems with friction," IEEE Transactions on Automatic Control, vol. 40, no. 3, pp. 419-425, 1995.

[15] P. Dupont, V. Hayward, B. S. R. Armstrong, and F. Altpeter, "Single state elastoplastic friction models," IEEE Transactions on Automatic Control, vol. 47, no. 5, pp. 787-792, 2002.

[16] J. Lin and C. H. Chen, "Positioning and tracking of a linear motion stage with friction compensation by fuzzy logic approach," ISA Transactions, vol. 46, no. 3, pp. 327-342, 2007.

[17] H. T. Yau and J. J. Yan, "Adaptive sliding mode control of a high-precision ball-screw-driven stage," Nonlinear Analysis: Real World Applications, vol. 10, no. 3, pp. 1480-1489, 2009.

[18] S. I. Han and K. S. Lee, "Robust friction state observer and recurrent fuzzy neural network design for dynamic friction compensation with backstepping control," Mechatronics, vol. 20, no. 3, pp. 384-401, 2010.

[19] S. H. Park and S. I. Han, "Robust-tracking control for robot manipulator with deadzone and friction using backstepping and RFNN controller," IET Control Theory \& Applications, vol. 5, no. 12, pp. 1397-1417, 2011.

[20] F. L. Lewis, W. K. Tim, L. Z. Wang, and Z. X. Li, "Deadzone compensation in motion control systems using adaptive fuzzy logic control," IEEE Transactions on Control Systems Technology, vol. 7, no. 6, pp. 731-742, 1999.

[21] X. S. Wang, C. Y. Su, and H. Hong, "Robust adaptive control of a class of nonlinear systems with unknown dead-zone," Automatica, vol. 40, no. 3, pp. 407-413, 2004.

[22] Z. Wang, Y. Zhang, and H. Fang, "Neural adaptive control for a class of nonlinear systems with unknown deadzone," Neural Computing and Applications, vol. 17, no. 4, pp. 339-345, 2008.

[23] W. M. Bessa, M. S. Dutra, and E. Kreuzer, "Sliding mode control with adaptive fuzzy dead-zone compensation of an electrohydraulic servo-system," Journal of Intelligent and Robotic Systems: Theory and Applications, vol. 58, no. 1, pp. 3-16, 2010.

[24] L. X. Wang, "Stable adaptive fuzzy control of nonlinear systems," IEEE Transactions on Fuzzy Systems, vol. 1, no. 2, pp. 146-155, 1993.

[25] X. D. Zhao, L. X. Zhang, P. Shi, and H. R. Karimi, "Novel stability criteria for T-S fuzzy systems," IEEE Transactions on Fuzzy Systems, 2013. 
[26] Y. C. Chang, "Robust tracking control for nonlinear MIMO systems via fuzzy approaches," Automatica, vol. 36, no. 10, pp. 1535-1545, 2000.

[27] E. Kim, "Output feedback tracking control of robot manipulators with model uncertainty via adaptive fuzzy logic," IEEE Transactions on Fuzzy Systems, vol. 12, no. 3, pp. 368-378, 2004.

[28] J. P. Hwang and E. Kim, "Robust tracking control of an electrically driven robot: adaptive fuzzy logic approach," IEEE Transactions on Fuzzy Systems, vol. 14, no. 2, pp. 232-247, 2006.

[29] V. I. Utkin, Sliding Modes in Control and Optimization, Communications and Control Engineering Series, Springer, New York, NY, USA, 1992.

[30] J. E. Slotine and W. Li, Applied Nonlinear Control, Prentice Hall, Englewood Cliffs, NJ, USA, 1991.

[31] S. W. Kim and J. J. Lee, "Design of a fuzzy controller with fuzzy sliding surface," Fuzzy Sets and Systems, vol. 71, no. 3, pp. 359367, 1995.

[32] W. Y. Wang, M. L. Chan, C. C. J. Hsu, and T. T. Lee, " $H_{\infty}$ tracking-based sliding mode control for uncertain nonlinear systems via an adaptive fuzzy-neural approach," IEEE Transactions on Systems, Man, and Cybernetics B, vol. 32, no. 4, pp. 483492, 2002.

[33] J. C. Scarratt, A. S. I. Zinober, R. E. Mills, M. Rios-Bolívar, A. Ferrara, and L. Giacomini, "Dynamical adaptive first and second-order sliding backstepping control of nonlinear nontriangular uncertain systems," Journal of Dynamic Systems, Measurement and Control, vol. 122, no. 4, pp. 746-752, 2000.

[34] A. J. Koshkouei, A. S. I. Zinober, and K. J. Burnham, "Adaptive sliding mode backstepping control of nonlinear systems with unmatched uncertainty," Asian Journal of Control, vol. 6, no. 4, pp. 447-453, 2004.

[35] F. J. Lin, C. K. Chang, and P. K. Huang, "FPGA-based adaptive backstepping sliding-mode control for linear induction motor drive," IEEE Transactions on Power Electronics, vol. 22, no. 4, pp. 1222-1231, 2007.

[36] B. K. Yoo and W. C. Ham, "Adaptive control of robot manipulator using fuzzy compensator," IEEE Transactions on Fuzzy Systems, vol. 8, no. 2, pp. 186-199, 2000.

[37] R. J. Wai and P. C. Chen, "Intelligent tracking control for robot manipulator including actuator dynamics via TSK-type fuzzy neural network," IEEE Transactions on Fuzzy Systems, vol. 12, no. 4, pp. 552-559, 2004.

[38] L. Lu, B. Yao, Q. Wang, and Z. Chen, "Adaptive robust control of linear motors with dynamic friction compensation using modified LuGre model," Automatica, vol. 45, no. 12, pp. $2890-$ 2896, 2009. 


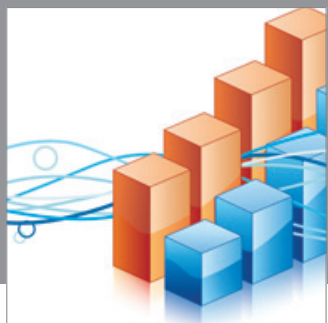

Advances in

Operations Research

mansans

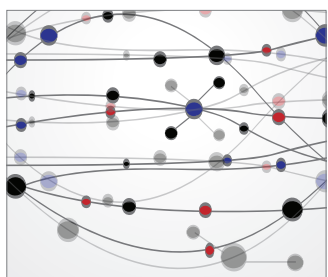

The Scientific World Journal
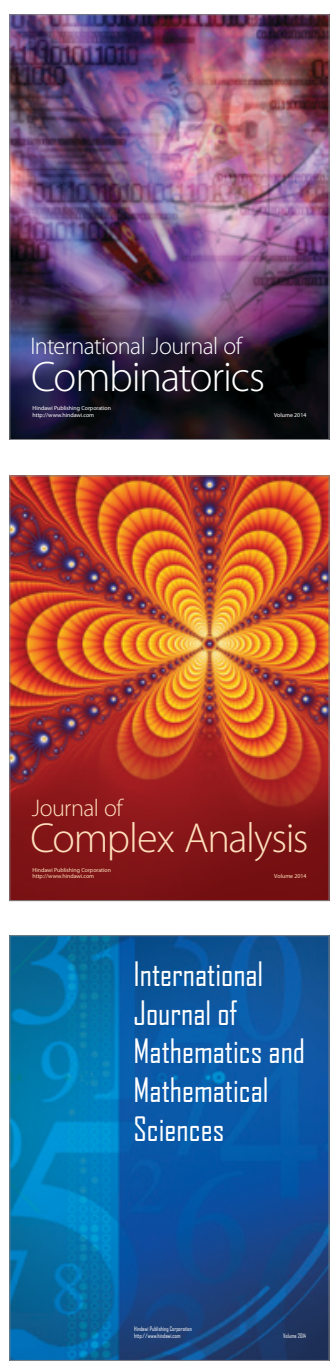
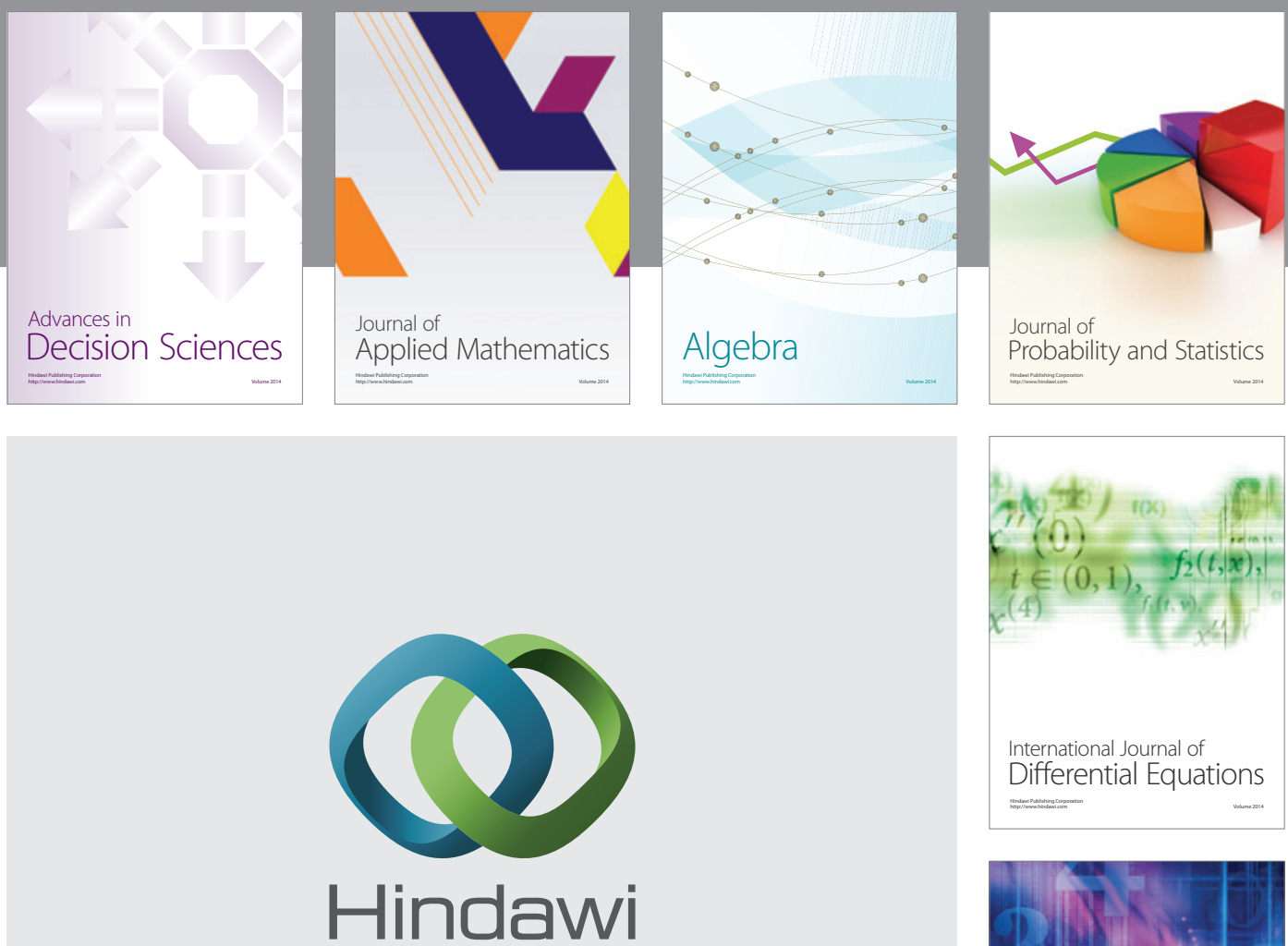

Submit your manuscripts at http://www.hindawi.com
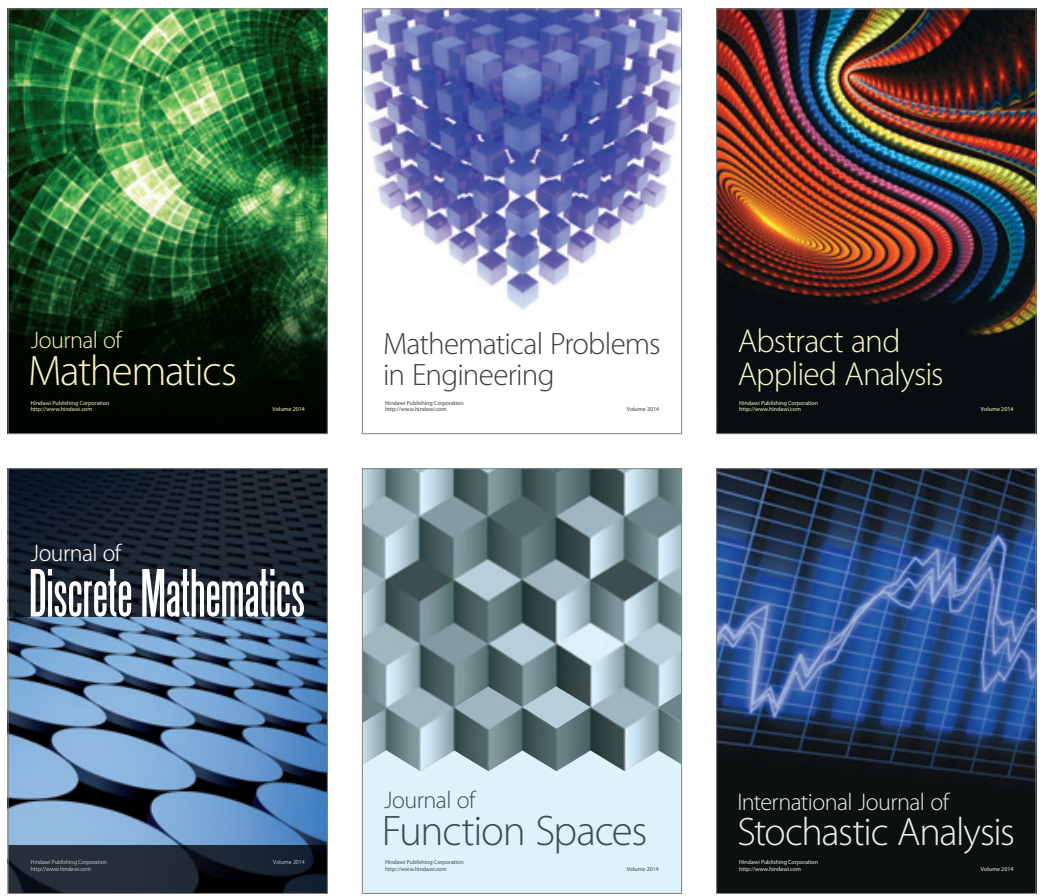

Journal of

Function Spaces

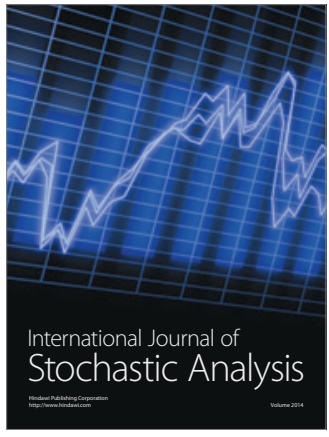

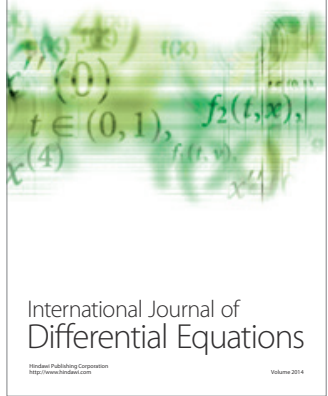
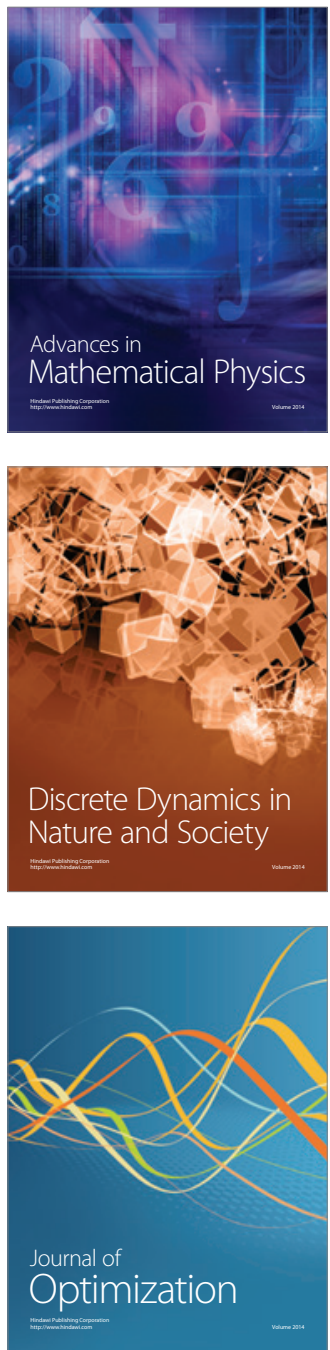